\title{
An automated search for compact high-velocity clouds in the Leiden/Dwingeloo Survey ${ }^{\star}$
}

\author{
V. de Heij ${ }^{1}$, R. Braun ${ }^{2}$, and W. B. Burton ${ }^{1,3}$ \\ 1 Sterrewacht Leiden, PO Box 9513, 2300 RA Leiden, The Netherlands \\ 2 Netherlands Foundation for Research in Astronomy, PO Box 2, 7990 AA Dwingeloo, The Netherlands \\ 3 National Radio Astronomy Observatory, 520 Edgemont Road, Charlottesville, Virginia 22903, USA
}

Received 20 June 2001/ Accepted 31 May 2002

\begin{abstract}
We describe an automated search through the Leiden/Dwingeloo H I Survey (LDS) for high-velocity clouds north of $\delta=-28^{\circ}$. From the general catalog we extract a sample of relatively small (less than about $8^{\circ}$ ) and isolated high-velocity clouds, CHVCs: anomalous-velocity H I clouds which are sharply bounded in angular extent with no kinematic or spatial connection to other $\mathrm{H}_{\mathrm{I}}$ features down to a limiting column density of $1.5 \times 10^{18} \mathrm{~cm}^{-2}$. This column density is an order of magnitude lower than the critical H I column density, $\sim 2 \times 10^{19} \mathrm{~cm}^{-2}$, (e.g. Maloney 1993) where the ionized fraction is thought to increase dramatically due to the extragalactic radiation field. As such, these objects are likely to provide their own shielding to ionizing radiation. Their small angular size, of less than about $1^{\circ} \mathrm{FWHM}$, might then imply substantial distances, since the partially ionized $\mathrm{H}_{\mathrm{I}}$ skin in a power-law ionizing photon field has a typical exponential scale-length of $1 \mathrm{kpc}$ (e.g. Corbelli \& Salpeter 1993). The automated search algorithm has been applied to the HIPASS and to the Leiden/Dwingeloo data sets. The results from the LDS are described here; Putman et al. (2002) describe application of this algorithm to the HIPASS material. We identify 67 CHVCs in the LDS which satisfy stringent requirements on isolation, and an additional 49 objects which satisfy somewhat less stringent requirements. Independent confirmation is available for all of these objects, either from earlier data in the literature or from new observations made with the Westerbork Synthesis Radio Telescope and reported here. The catalog includes 54 of the 65 CHVCs listed by Braun \& Burton (1999) on the basis of a visual search of the LDS data.
\end{abstract}

Key words. ISM: clouds - ISM: kinematics and dynamics - Galaxy: evolution - galaxies: dwarf - galaxies: evolution galaxies: Local Group

\section{Introduction}

High-velocity clouds (HVCs) were first encountered in the $\lambda 21 \mathrm{~cm}$ line of $\mathrm{H} \mathrm{I}$ at radial velocities unexplained by any conventional model of Galactic rotation. Since their discovery by Muller et al. (1963), they have remained enigmatic objects of continued interest. Wakker \& van Woerden (1997) and Wakker et al. (1999) have given recent reviews; since these reviews, progress has been made on several fronts. The anomalousvelocity clouds are found scattered over the entire sky, and examples are found throughout a range of radial velocity spanning about $800 \mathrm{~km} \mathrm{~s}^{-1}$ : obtaining an adequate observational foundation for the phenomenon has been a persistent and continuing challenge. We describe here a search algorithm which has been applied to the all-sky coverage afforded by the new

Send offprint requests to: $\mathrm{R}$. Braun,

e-mail: rbraun@astron.nl

* Table 1 is only available in electronic form at the CDS via anonymous ftp to cdsarc.u-strasbg.fr $(130.79 .125 .5)$ or via http://cdsarc.u-strasbg.fr/cgi-bin/qcat?J/A+A/391/159 Figures 6 to 9 are only available in electronic form at http://www. edpsciences.org
H I surveys of the northern and southern skys, and the results of its application to the Leiden/Dwingeloo Survey for examples of the phenomenon.

During the past forty years, a wide variety of explanations for the HVCs has been suggested. The matter of distances has remained particularly difficult, and the intrinsic physical properties derived from observational data depend critically on the distance. Only in a few cases have distances been measured or constrained. The distinct system of anomalous-velocity features recognised as the Magellanic Stream represents tidal debris originating in a gravitational interaction of the Large and Small Magellanic Clouds with our Galaxy (see Putman \& Gibson 1999), and is therefore likely to be located at distances of several tens of kpc. Other distinct systems of high-velocity objects constitute a few complexes, stretching over regions of some tens of square degrees. One of these, Complex A, has been found from absorption-line observations (van Woerden et al. 1999; Wakker 2001) to lie within the distance range $8<d<10 \mathrm{kpc}$. But the term HVC has been used to encompass a wide range of phenomena; unlike the Magellanic Stream and the half-dozen well-known complexes, many of the individual anomalous-velocity features are compact and are isolated on 
the sky down to low column density limits, as we will demonstrate below. The properties of anomalous-velocity H I emission might be more readily determined after a classification into sub-categories has been made. After compiling a general catalog of high-velocity features in the northern sky, we focus on identifying the category of compact, isolated features, which show no connection in position and velocity with the Galaxy, the Magellanic Clouds or the extended HVC complexes. Braun \& Burton (1999) have argued that these objects may represent a single class of clouds, whose members originated under similar circumstances and which share a common evolutionary history, and which might lie scattered throughout the Local Group.

The idea that the anomalous-velocity clouds are deployed throughout the Local Group has been considered earlier, by (among others) Oort (1966, 1970, and 1981), Verschuur (1975), Eichler (1976), Einasto et al. (1976), Giovanelli (1981), Arp (1985), and Bajaja et al. (1987). Various arguments have been raised against these interpretations. In the first review of the possible interpretations of high-velocity clouds, Oort (1966) ruled out the supposition that the clouds could be independent systems in the Local Group on two principal grounds: he stated that " ... a situation outside our Galaxy would give no explanation of the principal characteristic of the high-latitude clouds, viz. that the high velocities ... are all negative", and furthermore that " ... it would be almost impossible to explain on this hypothesis high-velocity clouds which apear to be related with each other over regions $30^{\circ}$ or more in diameter". Since Oort's first review, newer H I surveys have extended the sky coverage and have revealed that there are, in fact, approximately as many (compact) anomalous-velocity clouds at positive velocities as there are at negative velocities; and the objection against the large angular size of the complexes is confronted by the knowledge that these features, in any case, are indeed located within the Galactic halo.

By analyzing the stability of what they consider a representative HVC (in the Wakker \& van Woerden 1991 tabulation) against Galactic tidal disruption and self-gravity, Blitz et al. (1999) suggest a distance of $1 \mathrm{Mpc}$, for an assumed ratio between $\mathrm{H}_{\mathrm{I}}$ mass and total mass of 0.1 . Furthermore they suggest that the preferred coordinate system for the clouds is neither the Local Standard of Rest system, nor the Galactic Standard of Rest system, but the Local Group Standard of Rest system. The amplitude of the average velocity and the velocity dispersion of the cloud system both have the lowest values in this system, indicating that it might be the most relevant. However, the role of Galactic foreground obscuration has not yet been properly modeled to assess it's influence on these distributions. A numerical simulation of the dynamics of a population of low mass test masses within the gravitational potential of the Milky Way and M 31, reproduces some aspects of the kinematic and spatial distribution of the clouds. They suggest that the HVCs are the unused building blocks of the Local Group, falling towards its barycenter.

Braun \& Burton (1999, hereafter BB99) reached similar conclusions based on a study of a distinct subset of the HVC population. By restricting their attention to compact, isolated CHVCs, they exclude the contribution of the nearby, less representative clouds. The hypothesis is that the compact clouds might be the distant counterparts of the nearby, large angular size complexes. The compact sample also shows a natural preference for the Local Group Standard of Rest system, wherein its velocity dispersion $\left(88 \mathrm{~km} \mathrm{~s}^{-1}\right)$ is lower than in either the LSR or GSR frames. The CHVCs even allow definition of a new coordinate system in which a global minimum of the velocity dispersion $\left(69 \mathrm{~km} \mathrm{~s}^{-1}\right)$ is obtained. This system agrees with the Local Group system at about the one sigma level. Furthermore, analysis of high resolution images of sixteen of the CHVCs provide several independent, although indirect, indications of distances of between 150 and $850 \mathrm{kpc}$ (Braun \& Burton 2000; Burton et al. 2001).

The BB99 sample was obtained by visual inspection of the Leiden/Dwingeloo Survey (LDS) of the local H I sky carried out by Hartmann \& Burton (1997). The LDS surveyed the sky as far south as the Dwingeloo horizon, that is to a declination of $-30^{\circ}$; lacking information on the more southern declinations, the BB99 conclusions were based on an incomplete sample. A major improvement of the CHVC study would be an extension to the whole sky. Its high sensitivity and fullyNyquist sampling makes the recently completed Parkes AllSky Survey, HIPASS, (Barnes et al. 2001) ideal for extending the CHVC sample. To create an all-sky resource which is as homogeneous as possible, an automated algorithm has been developed and is described here. This paper also discusses application of the algorithm to the LDS; a separate paper (Putman et al. 2002) gives the results from applying the algorithm to the HIPASS southern-hemisphere data.

Our discussion is organized as follows. We begin by describing the data used and the importance of obtaining confirming observations in Sect. 2, proceed with a description of the algorithm and selection criteria in Sect. 3, present a catalog of both compact and extended high-velocity clouds in Sect. 4, and conclude with a brief discussion of the global properties of the cataloged objects in Sect. 5.

\section{Observations}

The LDS was observed with the 25-m Dwingeloo telescope, whose $F W H M$ beam subtends 36 arcmin, on a grid of 0.5 by 0.5 true-angle separation. It covered the sky north of declination $-30^{\circ}$ completely on this grid, and extended in a less complete fashion a few degrees further south. The effective velocity coverage of the LDS spans Local Standard of Rest velocities from $-450 \mathrm{~km} \mathrm{~s}^{-1}$ to $+400 \mathrm{~km} \mathrm{~s}^{-1}$, resolved into spectral channels of $1.03 \mathrm{~km} \mathrm{~s}^{-1}$ width. The nominal brightness-temperature sensitivity of the LDS is $0.07 \mathrm{~K}$, although this value varies for individual spectra. Hartmann et al. (1996) describe the corrections applied to the LDS material in order to remove contamination by stray radiation.

There can be various causes of imperfections in a survey such as the Leiden/Dwingeloo one; of these, radio frequency interference (RFI) is the most pernicious, as it can be responsible for false detections of just the sort of spectral signals being sought in this analysis, namely features of quite weak intensity, moderate-to-substantial frequency width, and occuring in only one spectrum or in a few adjacent spectra. More commonly, RFI produces extremely narrow spike signals, or a 
characteristic $\sin (x) / x$ ringing, and can be rather easily recognised by these and other properties as not being of an interstellar origin. But Hartmann (1994) and Hartmann \& Burton (1997) show examples of RFI signals detected in the LDS which mimic the properties of spectral features of astronomical interest. In these examples, the interference was of short temporal duration, thereby disabling the common diagnostic tool of being on the look-out for features remaining at a constant frequency, or with an unusual telltale drift in frequency.

Because the isolated objects being sought here appear at only several of the LDS lattice points, or even at only one, confirmation was sought by independent observations. This policy of demanding independent confirmation in all cases had lead BB99 to reject many candidate CHVCs from their listing. BB99 had been able to carry out the independent confirmations using either the NRAO 140-foot telescope or the Dwingeloo 25-meter; but since neither of these instruments is currently operative, we sought confirmations in new observations, made with the Westerbork Synthesis Radio Telescope (WSRT). The importance of the confirmation observations is stressed by the fact that only 116 of the 171 candidate isolated features which we found in the LDS could be verified. Some candidates, like the one shown in Fig. 1, were revealed to be due to RFI contamination and thus spurious, despite the fact that their spectral properties show similarities with those of a genuine astronomical feature. Other candidates masquerading as astronomical features were attributed to the vagaries of noise. (Knowing that confirmation would be demanded, the original list of 171 candidates was prepared with rather liberal noise constraints.)

Confirming observations were deemed unnecessary for those candidate features which could be identified with objects listed in previously published investigations based on independent data. Most important in this regard are the high-velocity cloud catalogs of Wakker \& van Woerden (1991), extracted from the surveys of Hulsbosch \& Wakker (1988) and Bajaja et al. (1985); the BB99 catalog of CHVCs extracted from the Leiden/Dwingeloo survey and reconfirmed with additional observations; and the catalog of Putman et al. (2002) extracted from the HIPASS data using the algorithm described here.

The differences in angular and velocity resolutions, sampling intervals, and sensitivities of the surveys required different criteria to ascertain matches with the features identified as candidates by the search algorithm. Identification of the candidates with the BB99 objects is straightforward, because the LDS material serves as input in both cases; no additional confirmation was required, because BB99 had already adequately confirmed the signals. Establishing correspondence with objects in the Putman et al. catalog is also straightforward. The HIPASS material was Nyquist sampled at the angular resolution of 15.5 afforded by the Parkes $64-\mathrm{m}$ telescope, and at a $5 \sigma$ rms brightnes-temperature sensitivity of approximately $50 \mathrm{mK}$ over $26 \mathrm{~km} \mathrm{~s}^{-1}$. In these observational parameters the HIPASS data surpasses the LDS data, and they suffice as independent confirmation of candidates identified in the LDS. We note, however, that the $26 \mathrm{~km} \mathrm{~s}^{-1}$ velocity resolution of the HIPASS data is substantially coarser than
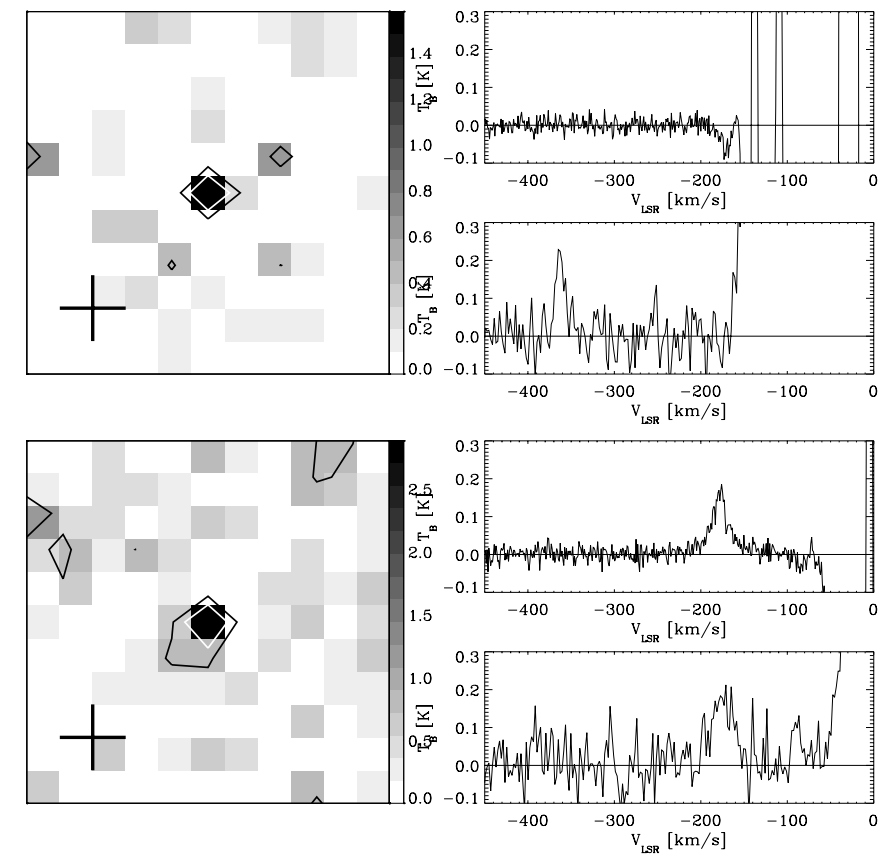

Fig. 1. Two examples illustrating the role played by independent confirming observations made using the Westerbork Synthesis Radio Telescope in total-power mode. Each triplet of panels shows the LDS sky image of a candidate, a spectrum from the LDS (lower spectrum) and a spectrum from the new WSRT observations (upper spectrum). The upper candidate, CHVC 099+07-356, could not be confirmed; the lower candidate, CHVC 015-05-171 could. The WSRT spectra used for the confirmation of all LDS candidates for which there were no other independent data were substantially more sensitive than the LDS material. The crosses on the lower left of the sky images show the angular extent of a true degree on the sky. Contours are drawn in these images at $50 \%$ and $25 \%$ of the peak value of the signal perceived from the candidate cloud; the gray color-bar indicates scaling in units of $\mathrm{K} \mathrm{km} \mathrm{s}^{-1}$.

the $1.03 \mathrm{~km} \mathrm{~s}^{-1}$ resolution of the LDS; thus it is possible that an object of narrow linewidth would be detected in the LDS but would be diluted by as much as a factor of 25 in the coarser HIPASS velocity coverage. There are, in fact, two objects listed in the CHVC catalog given in Table 2 which lie at declinations in the overlap zone, $-30^{\circ}<\delta<+2^{\circ}$, but which are not listed in the Putman et al. catalog.

Establishing correspondence with objects listed in the Wakker \& van Woerden (1991) catalog is less straightforward, because that listing is based on $\mathrm{H}_{\mathrm{I}}$ observations made at substantially coarser angular and spectral sampling than pertain to the LDS (although at comparable sensitivity, if measured after the surveys are convolved to similar spectral and angular resolutions). The Hulsbosch \& Wakker (1988) data, on which most of the Wakker \& van Woerden catalog depends, pertains to observations made using the Dwingeloo 25-meter telescope at declinations above $-18^{\circ}$, at a velocity resolution of $16 \mathrm{~km} \mathrm{~s}^{-1}$, and on a $1^{\circ}$ by $1^{\circ}$ grid; the southern material of Bajaja et al. (1985) was observed on the IAR 100-foot telescope at somewhat lower rms sensitivity then the Hulsbosch \& Wakker survey and at comparable velocity resolution, but on a coarser initial sampling grid of $2^{\circ}$ by $2^{\circ}$, supplemented by finer sampling in many cases. 
Candidates identified by our search algorithm in the LDS were judged to correspond with objects in the catalog of Wakker \& van Woerden if the spatial separation of the candidate and the Wakker \& van Woerden listing does not exceed $1^{\circ}$ and if, in addition, the velocity separation does not exceed $25 \mathrm{~km} \mathrm{~s}^{-1}$; furthermore, the velocity difference was required to be less than the velocity dispersion of the Gaussian which fits the central spectrum: the clouds in the Wakker \& van Woerden catalog were constructed from the components listed by Hulsbosch \& Wakker. A correspondence between the profile components and the LDS candidate which met these criteria was judged as independent confirmation. Of all of the moderately isolated objects cataloged in Table 2, i.e. including the unambiguous CHVCs, as well as the partially confused CHVC:, and CHVC? categories defined in detail below, 33 of the 116 could not be identified with a Wakker \& van Woerden cloud; 28 of the 67 objects classified as CHVCs do not have a Wakker \& van Woerden counterpart. In all of the cases where there is an identifiable counterpart, the coarser resolution of the Wakker \& van Woerden material precludes measuring the degree of compactness or isolation: these measures in Table 2 are based on the LDS data.

For the confirming observations which we required for all of the candidates with no definite counterpart in earlier data, we used the Westerbork Synthesis Radio Telescope in the newly-available total-power observing mode whereby high-resolution auto-correlation spectra are obtained from all 14 individual 25-meter antennas, rather than the more usual cross-correlation spectra. The position-switching mode involved observing oN-spectra with the antennas pointed toward the candidate and ofF-spectra pointing at the same declination but with right ascension offsets of $\pm 3^{\circ}$. The data from all 14 different telescopes and both linear polarisations were averaged into a single spectrum, after obvious interference signals and other forms of unreliable data had been removed. Finally, the (ON-OFF)/ OFF spectrum was determined, using the average of both off spectra. With an average rms noise of $0.02 \mathrm{~K}$ over $1.03 \mathrm{~km} \mathrm{~s}^{-1}$, the WSRT spectra are substantially more sensitive than the original Leiden/Dwingeloo spectra for which confirmation was being sought. Figure 1 displays two WSRT and LDS pairs, one of which provided confirmation and one of which revealed RFI contamination perniciously mimicking a compact high-velocity cloud.

\section{Algorithm and selection criteria}

\subsection{Algorithm}

A quantified, automated routine for extracting HVCs should be designed such that it can be applied in a general way, i.e. to surveys other than the LDS; in that way it can also permit analysis of sample completeness. There are several different options for extracting structure from a three-dimensional data cube, each having certain advantages and disadvantages. By using a predefined cloud model, for example, one could decompose the data into a set of clouds which conform to that predefined model. The input cloud model can be described by a parametric function which is subsequently fit to the data. Because the shape of each cloud is presumed known, one is able, in the context of that presumption, to handle blended emission from two or more clouds. An example of this approach is given by Stutzki \& Güsten (1990), who used a Gaussian parametric form to unravel $\mathrm{C}^{18} \mathrm{O}$ emission from molecular clouds. Alternatively, one could create a predefined set of various possible clouds. Thilker et al. (1998) designed such an algorithm, and applied it to look for $\mathrm{H}_{\mathrm{I}}$ bubbles blown by supernovae in external galaxies.

A different approach, not based on an a priori cloud model, was used by Williams et al. (1994), among others. Williams et al. defined a set of contours of constant intensity, and then scanned their molecular-cloud data for clumpy structure. Starting at a high intensity level, a closed contour only contains the peak of a clump; by slowly decreasing the contour level, the exact shape of the cloud emerges. If the emission of a nearby cloud shows up in the contour, a friend-of-friend algorithm can be used to determine to which cloud each pixel belongs. To extract clouds properly, the difference between adjacent contour levels has to be small: otherwise, two nearby clouds which each contribute local intensity peaks but with only small differences in the peak values will be extracted as a single structure.

Following the Williams et al. (1994) approach, we also use a procedure without a presumed cloud model. But in our approach, instead of scanning for clouds along contours of constant intensity at varying levels, we use the gradient of the intensity field to determine the structure to which the pixels should be assigned. By assuming that the pixels belong to the same structure as their brightest neighbours, we are able to extract clouds of arbitrary shapes and sizes.

The procedure is illustrated by Fig. 2. Starting at any pixel, we proceed to its brightest neighbour, and then keep continuously moving to the brightest neighbour of each pixel we pass, until a local maximum is found. The local maximum defines the peak of the structure of which the complete track followed is part. Applying this procedure to all pixels enables us to split up the complete three-dimensional data set into structures, i.e. clouds. The exact result depends on which of the adjacent pixels are called neighbours. One could confine the set of neighbours to the adjacent pixels with a difference in one, two, or three coordinate values of the three-dimensional set. During our search for isolated high-velocity clouds, we treated all adjacent pixels as neighbours, which are 26 in number for a threedimensional data set.

Each local maximum in the data is, formally, the peak of a cloud, even if the local maximum is just a small wiggle in a spectrum produced, for example, by the vagaries of Gaussian noise. To reduce the effect of local maxima with a low contrast compared to their environment, adjacent clouds with low contrast were merged: specifically, two adjacent clouds were merged if the level of the brightest intensity contour which enclosed the peaks of both clouds exceeded either $0.2 \mathrm{~K}$ or $50 \%$ of the intensity of the brightest peak. The brighter of the peaks of the merged structures was considered the peak of the new cloud. If a cloud was encompassed by more than one neighbour, it was joined with the neighbour with the lowest contrast. Once a new cloud was formed, we checked if neighbouring structures should then be merged, according to the criterion described, with this new cloud. 

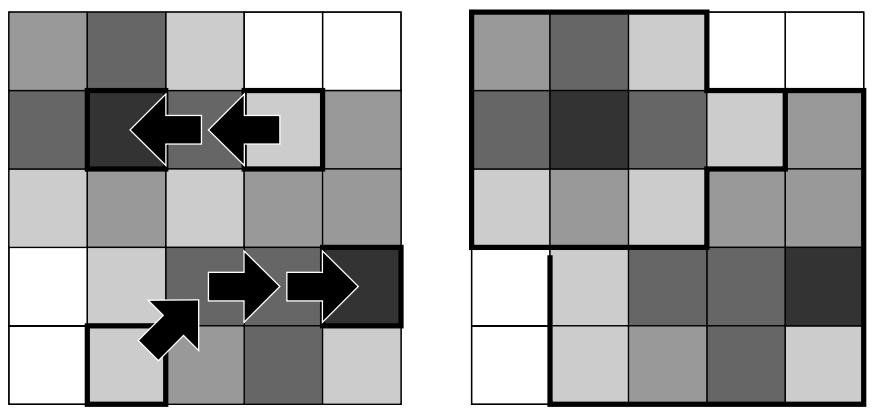

Fig. 2. Example in two dimensions illustrating the assignment of pixels to local maxima and the resulting definition of clouds. Starting at the pixels at low intensities (but above the $1.5 \sigma$ level), the algorithm finds the appropriate maximum by moving from brightest neighbour to brightest neighbour. Should a pixel have two equally bright neighbours, the assignment is random. The righthand panel shows the result of the pixel assignment.

Not all pixels were assigned to a cloud. We only assigned pixels with a signal-to-noise ratio of at least 1.5 to a cloud. Before the clouds were merged, we required that the intensities of their peaks should exceed three times the noise value. After all clouds were merged, we required furthermore that the peaks have a signal-to-noise ratio of at least five. As a consequence, a cloud candidate with a signal-to-noise ratio of three which is not merged with other clouds was removed from the list. To determine the correct intensities for the pixels which are formed into clouds and the peak intensities of the clouds before merging, we used a fixed, preset noise value valid for the complete survey. The signal-to-noise ratio of the peaks of the clouds after merging was determined from a locallymeasured noise value. This value was determined in a square measuring 31 by 31 pixels, centered on the cloud and located in the velocity channel map containing the peak intensity of the cloud in question. A lower limit was used for this newly determined noise value, equal to the preset noise value.

An iterative procedure was used to determine the noise in the region around the cloud peak. We started with a sample consisting of all pixels in the $31-$ by-31 pixel square. After determining the median, $\mu$, and the absolute deviation, $\delta$, of the pixels considered, all pixels which were not in the range $\mu \pm f \cdot \delta$ were removed from the sample. By repeatedly applying the rejection criterion, we created a sample for which all pixels lay in the range $\mu \pm f \cdot \delta$. The noise was then set equal to the standard deviation of this sample. The exact result depends heavily on the chosen value of the factor $f$. The more real emission there is in the sample, the lower the value of $f$ should be. We have used the value $f=3.0$, which was found to give reasonable results.

\subsection{Application of the search algorithm to the LDS}

The algorithm described above can be applied generally, i.e. to a variety of data sets. We describe here its application to the Leiden/Dwingeloo H I survey. The LDS was prepared for the algorithm as follows. It was first divided into 24 separate data cubes, each cube spanning an area of extent $128^{\circ}$ by $128^{\circ}$, and representing the $\mathrm{H}_{\mathrm{I}}$ sky in a zenith equal-area projection. The sky area beyond the inner $64^{\circ}$ by $64^{\circ}$ overlaps with neighbouring cubes. We constructed separate cubes for the positive and for the negative Local Standard of Rest velocities.

For the initial pass of the algorithm, the data cubes were Hanning smoothed, with the twofold motivation of reducing the detections of apparent clouds contributed by local maxima in the noise fluctuations and in order to reduce the amount of computer memory required to a level consistent with our capabilities. The data were smoothed with a Gaussian function with a $F W H M$ of $12 \mathrm{~km} \mathrm{~s}^{-1}$ in the spectral direction and 1.2 along the spatial axes. A constant rms noise value of $0.01 \mathrm{~K}$ was used for the preset noise parameter. Once all pixels with a sufficient brightness temperature were assigned to clouds, the Hanning smoothed data (angular sampling 0.5, velocity resolution $2.06 \mathrm{~km} \mathrm{~s}^{-1}$ ) were used to derive the cloud properties.

A velocity-integrated intensity map of each cloud was also extracted from the data. The range of integration extends over the velocity range of the pixels that were assigned to a particular cloud. A description of all derived cloud parameters is listed in Section 4. After lists of clouds and their properties were produced for all of the separate cubes, they were merged into one catalog. During the merging process double entries which were found in the regions of overlapping cubes were eliminated.

Initial application of the search algorithm to the LDS resulted in a list of all objects satisfying the search criteria, and thus included not only compact high-velocity clouds, but also structures that are part of the high-velocity-cloud complexes, the intermediate-velocity features, and even the gaseous disk of our Galaxy, as well as features which were subsequently eliminated as due to excessive noise, radio interference, the non-square response of the receiver bandpass, or other imperfections in the data. To remove emission associated with our Galaxy and the intermediate-velocity complexes, all clouds with a deviation velocity $V_{\mathrm{DEV}}$ less than $70 \mathrm{~km} \mathrm{~s}^{-1}$ were removed. The deviation velocity, as defined by Wakker (1990), is the excess velocity of a feature compared to the velocities allowed by a simple model of the kinematics of our Galaxy. A description of the model used here to define the deviation velocity is given in the following subsection.

In order to remove the putative clouds associated with imperfections in the data, an additional signal-to-noise criterion was adopted, namely that the line integral of the cloud in the spectrum which passes through the cloud peak should exceed the $8 \sigma$ value. To determine the noise in the spectrum, all channels identified with the feature and with a deviation velocity less than $100 \mathrm{~km} \mathrm{~s}^{-1}$ were excluded from consideration. The iterative procedure described in the previous section was then followed to determine $\sigma$. Features with $\int I \mathrm{~d} V /\left(\sqrt{n}_{\mathrm{V}} \cdot \sigma \cdot \Delta V\right)<$ 8 , (where $n_{\mathrm{V}}$ is the number of summed velocity channels of width $\Delta V$ ) were removed from the list.

The low-frequency edge of the receiver bandpass used in the LDS had a strong roll-off beyond about $+400 \mathrm{~km} \mathrm{~s}^{-1}$, which resulted in the occasional appearance of spectral features at the positive-velocity extremes which could be confused with one wing of a cloud of astronomical interest. The situation at the extreme positive velocities in the LDS is further confused by the relatively frequent occurance of interference in this regime. 
Although the positive-velocity wings of the LDS spectra published by Hartmann \& Burton (1997) had already been truncated at a $V_{\mathrm{LSR}}$ of $+400 \mathrm{~km} \mathrm{~s}^{-1}$, in recognition of the bandpass and RFI problems (even though the nominal response of the receiver extended to $+500 \mathrm{~km} \mathrm{~s}^{-1}$ ), it seemed practical to adopt an even lower upper-velocity limit for this project. Therefore all objects with $V_{\text {LSR }}$ greater than $+350 \mathrm{~km} \mathrm{~s}^{-1}$ were excluded from the list. This selection is, however, probably without any consequence for the high-velocity-cloud phenomenon. The most extreme positive velocity of the CHVC ensemble found by BB99 in the northern hemisphere was $+216 \mathrm{~km} \mathrm{~s}^{-1}$; the most extreme positive velocity found in this analysis is +268 , namely for CHVC: $357.5+05.6+268$. The southern hemisphere CHVC listing is particularly relevant in this regard; a deployment of objects scattered throughout the Local Group and with a net infall motion (see BB99) would result in more objects at positive velocities in the southern hemisphere than in the northern. Nevertheless, although relatively more positive-velocity CHVCs were indeed found by the Putman et al. (2002) search through the HIPASS material (using the algorithm described here) over the range $-700<V_{\mathrm{LSR}}<+500 \mathrm{~km} \mathrm{~s}^{-1}$, only one of the objects identified as a CHVC had a positive velocity more extreme than $+350 \mathrm{~km} \mathrm{~s}^{-1}$, namely CHVC $258.2-23.9+359$.

The LDS sampled the sky on the complete $0.5 \times 0.5$ grid down to a declination of $-30^{\circ}$, with some observations on an incomplete grid extending several degrees further south. The degree of isolation of an object can only be determined if the surroundings are well observed. Because the information on the surroundings could not be determined close to the edges of the survey coverage, no new anomalous-velocity object with a declination less than $-28^{\circ}$ was entered in the catalog as a CHVC. (Four entries in Table 2 have $\delta<-28^{\circ}$ : numbers 107 and 109 correspond to two objects discussed by BB99 - their numbers 56 and 58, respectively - and these had been subject by BB99 to new Dwingeloo observations on a Nyquist grid, confirming their classification; number 113 is confirmed by other data as indicated in Table 2; and number 114 lies in a region where the LDS is complete, despite the low declination.)

The degree of isolation of the clouds was determined from the velocity-integrated images covering an area measuring $10^{\circ}$ by $10^{\circ}$ centered on the position and velocity of the cloud under consideration. The velocity interval of each image was matched to the entire velocity extent of the object in question. An ellipse was fit to the contour with a value of half the maximum brightness of the cloud to allow tabulation of size and orientation. The degree of isolation is assessed on the basis of the lowest significant contour level of $\mathrm{H}_{\mathrm{I}}$ column density commensurate with the data sensitivity. Given the median FWHM linewidth of about $25 \mathrm{~km} \mathrm{~s}^{-1}$, this corresponds to a $3 \sigma$ level of about $1.5 \times 10^{18} \mathrm{~cm}^{-2}$. Although there is a small variation of the $3 \sigma N_{\mathrm{HI}}$ level with object linewidth, we chose to keep this value fixed for the purposes of uniformity. We demanded that this contour satisfy the following criteria: (1) that it be closed, with its greatest radial extent less than the $10^{\circ}$ by $10^{\circ} \mathrm{im}-$ age size; and (2) that it not be confused by the presence of adjacent extended emission. In practice, the primary criterion of demanding a closed contour at our cut-off column density was often sufficent to unambiguously select isolated objects.
Some ambiguity arose when additional diffuse emission (at a level near the cut-off) was present within the $10^{\circ}$ by $10^{\circ}$ field of the velocity integrated images. Such diffuse emission might either be physically associated with the object in question or simply be a confusing foreground or background component. Rather than rejecting all such ambiguous objects outright, the more promising candidates have been retained but were given another designation. Only unambiguous features are designated CHVCs. Objects with additional diffuse emission components in the field were designated either CHVC:s in the event of some elongation in the same sense as the background or CHVC?s in the event of a smooth, but elevated, background.

A slightly different criterion for isolation was employed by Putman et al. (2002) in their analysis of the HIPASS sample of HVCs. Rather than employing a fixed minimum column density contour to make this assessment, they employed the contour at $25 \%$ of the peak $N_{\mathrm{HI}}$ for each object. Since the majority of detected objects are relatively faint, with a peak column density near $12 \sigma$, the two criteria are nearly identical for most objects. Only for the brightest $\sim 10 \%$ of sources might the resulting classifications differ.

Figure 3 shows an example for each of the groups of the classification. Since a degree of subjectivity is involved in the selection of the most promising cadidates for the CHVC: and CHVC? designation, two of the authors ( $\mathrm{VdH}$ and $\mathrm{RB}$ ) each independently carried out a complete classification of the 1280 cloud candidates. Identical independent classifications were made in $89 \%$ of all cases. A consensus was reached for the remaining sources after re-examination.

\subsection{Deviation velocity}

Every direction on the sky contains some $\mathrm{H}_{\mathrm{I}}$ emission from the Milky Way, which needs to be avoided when searching for high-velocity clouds. The kinematic and spatial properties of the Milky Way are well-enough behaved, and well-enough known, that contamination of the anomalous-velocity sky by H I emission from the conventional Galactic disk can be largely avoided. Wakker (1990) introduced the measure of deviation velocity, defined as the difference between the velocity of the cloud and the nearest limit of the velocities allowed by a conventional model of the differential rotation of the Galaxy in the same direction. Wakker's definition of deviation velocity was based on the kinematic limits predicted for a differentially rotating flat disk of uniform thickness. In order to constrain possible Milky Way contamination more accurately, we modified the definition of deviation velocity to account for the fact that the Milky Way H I disk is warped, and also flares, i.e. increases in thickness with increasing Galactocentric distance, and that it is not circular when viewed from above, but lopsided.

In order to determine the $V_{\mathrm{LSR}}$ which corresponds to the given $V_{\mathrm{DEV}}$, we modeled the kinematics and spatial properties of the Galactic Hi and calculated synthetic Hi spectra. The range of acceptable Galactic velocities was then set by the velocities for which the brightness temperatures of the synthetic spectra, corresponding to this model, exceed $0.5 \mathrm{~K}$. The model consists of a thin disk which has constant properties (central 

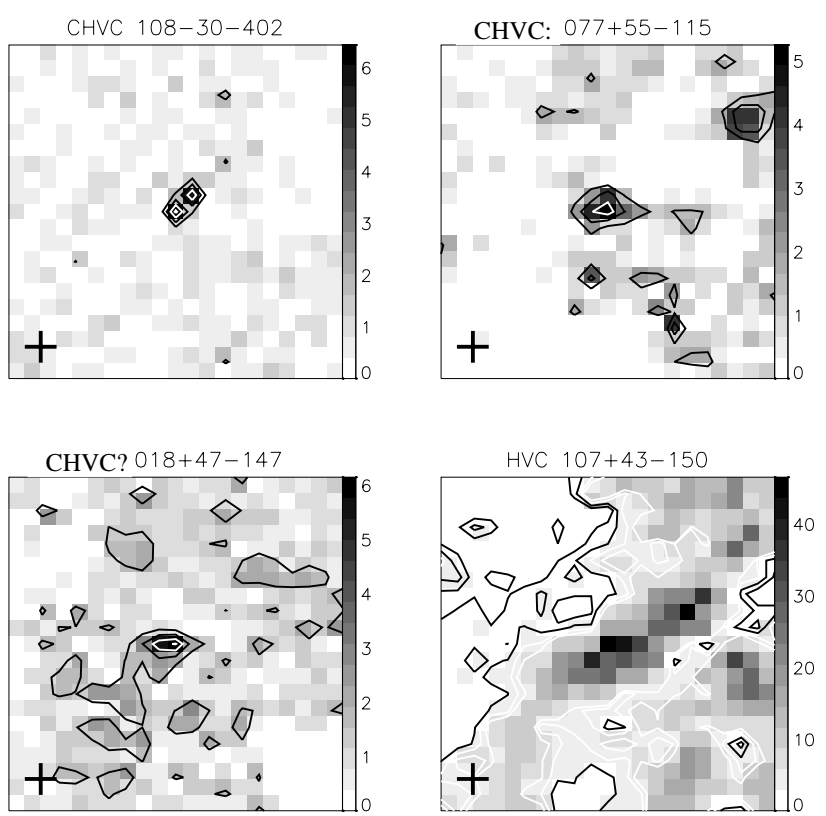

Fig. 3. Representative examples of the designation of confirmed anomalous-velocity features into the classes of CHVC, CHVC:, CHVC?, and HVC. The crosses on the lower left of each image show the angular extent of a true degree on the sky. The contours correspond to $N_{\mathrm{HI}}=1.5,3,4.5$ and $6 \times 10^{18} \mathrm{~cm}^{-2}$; the gray-scale-bar indicates scaling in units of $\mathrm{K} \mathrm{km} \mathrm{s}^{-1}$. Anomalous-velocity $\mathrm{H}_{\mathrm{I}}$ features designated as $\mathrm{CHVCs}$ are tightly constrained in their degree of isolation at $N_{\mathrm{HI}}=1.5 \times 10^{18} \mathrm{~cm}^{-2}$; CHVC:s have some elongation with respect to their environment; CHVC?s have an enhanced background $N_{\mathrm{HI}}$; and HVCs are organized into large complexes at a significantly higher $N_{\mathrm{HI}}=5-20 \times 10^{18} \mathrm{~cm}^{-2}$.

density, $n_{\mathrm{HI}}=0.35 \mathrm{~cm}^{-3}$, and kinetic temperature, $T_{k}=100 \mathrm{~K}$ ) within $11.5 \mathrm{kpc}$ from the Galactic center and which warps and flares beyond that radius. The vertical $z$-distribution of the gas layer is given by a Gaussian, with a dispersion of $180 \mathrm{pc}$ for $R \leq 11.5 \mathrm{kpc}$ and increasing by $80 \mathrm{pc}$ for each kpc further outward than $11.5 \mathrm{kpc}$. This thickness is higher than that derived by Baker \& Burton (1975), for example, in order account for the fact that the low-level wings of Galactic $\mathrm{H}_{\mathrm{I}}$ are generally broader than the Gaussian form exhibited at higher intensities, and thus to include more of the low-level disk gas, especially in the outer Galaxy, into the model. For $R \leq 11.5 \mathrm{kpc}$ the Gaussian is centered around $z=0 \mathrm{kpc}$; for $R>11.5 \mathrm{kpc}$, the center of the gas layer is at the height

$z=\frac{R-11.5}{6} \sin (\phi)+0.3\left(\frac{R-11.5}{6}\right)^{2}(1-2 \cos (\phi))$

as determined by Binney \& Merrifield (1998) from the Voskes $\&$ Burton (1999) analysis of combined southern and northern Milky Way H i survey data. The variable $\phi$ is the galactocentric cylindrical coordinate, which increases in the direction of Galactic rotation and equals $180^{\circ}$ towards the Sun. We included an exponential, radial decrease in density, with scale length $3.0 \mathrm{kpc}$, for $R>11.5 \mathrm{kpc}$, in order to improve the resemblance between the model and data. The Sun is located at $8.5 \mathrm{kpc}$ from the Galactic center; the gas follows circular rotation with a flat
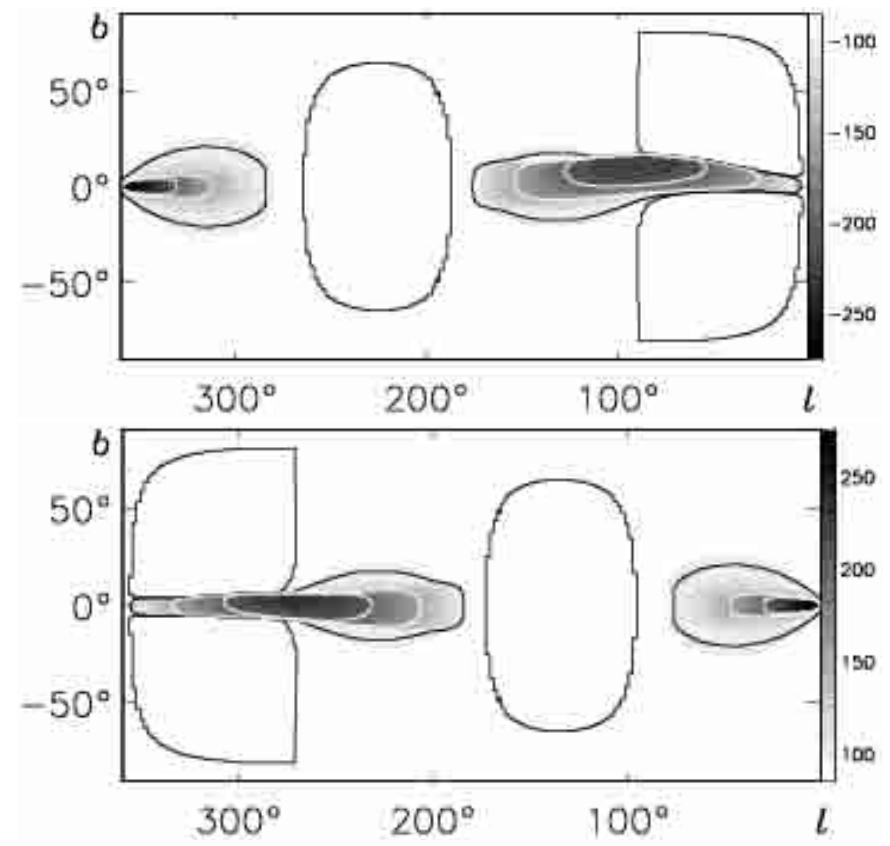

Fig. 4. Velocities excluded from the search procedure, because of possible contamination by $\mathrm{H}_{\mathrm{I}}$ emission from the Milky Way gaseous layer. The deviation velocity was defined to be $70 \mathrm{~km} \mathrm{~s}^{-1}$ more extreme than the empirically determined extrema of the conventional gaseous layer, albeit warped and lopsided (Voskes \& Burton 1999). The $V_{\mathrm{LSR}}$ which corresponds with a $V_{\mathrm{DEV}}$ of $70 \mathrm{~km} \mathrm{~s}^{-1}$ is shown as function of the Galactic longitude and latitude. For each line of sight there is a negative (top) and a positive $V_{\mathrm{LSR}}$ (bottom) corresponding with the given $V_{\mathrm{DEV}}$. Black contours are drawn at \pm 90 and $\pm 100 \mathrm{~km} \mathrm{~s}^{-1}$; white ones, at \pm 150 and $\pm 200 \mathrm{~km} \mathrm{~s}^{-1}$.

rotation curve at the level $220 \mathrm{~km} \mathrm{~s}^{-1}$. Furthermore, the synthetic H I spectra were broadened with a Gaussian distribution with a dispersion of $20 \mathrm{~km} \mathrm{~s}^{-1}$, in order to allow, conservatively, for the somewhat ragged edge of the outer Milky Way, observed at low latitudes, and for the filamentary intermediatevelocity clouds, commonly observed at higher latitudes.

Figure 4 shows the limiting $V_{\mathrm{LSR}}$ corresponding to a $V_{\mathrm{DEV}}$ of $70 \mathrm{~km} \mathrm{~s}^{-1}$ for this model, as function of Galactic longitude and latitude. The deviation-velocity approach was adopted in order to eliminate contamination by the conventional Galactic disk, although most models of the anomalous-velocity clouds allow these objects to pervade velocities near zero $\mathrm{km} \mathrm{s}^{-1}$. The deviation-velocity approach prejudices against detecting CHVCs at low velocities, but we note that they would likely remain unrecognized at low velocites in any case, because of the dominance of the ubiquitous Milky Way H I emission.

\section{Results}

\subsection{Catalog of high-velocity clouds}

Table 1 lists all 917 HVCs found in the LDS with our algorithm. Due to the extent of the table it is only available electronically and not in the printed version of this paper. Only the first 15 columns (as defined below) are included for this table. The reader should be aware that not all entries have been independently confirmed (see the Col. 15 description below). 
The subset of 116 objects which at least partially fulfill the criteria for source isolation and have been confirmed in independent observations are listed in Table 2. In addition to the welldefined CHVCs, the more ambiguous CHVC:s and CHVC?s, likewise independently confirmed, but satisfying less stringent criteria of apparent isolation are also included in the table. As discussed above, features designated with CHVC: or CHVC? had some ambiguity in their degree of isolation as ascertained with the LDS data. Some specific shortcomings of the CHVC? candidates are indicated by notes in the table; these include not satisfying the $V_{\mathrm{DEV}}>70 \mathrm{~km} \mathrm{~s}^{-1}$ criterion, lying near the edge of the LDS survey coverage either spatially or in velocity and in some cases the presence of a significant background level.

The columns of the table denote the following:

Column 1: Running identifying number in the catalog.

Column 2: Designation, consisiting of a prefix, followed by the Galactic longitude, Galactic latitude, and Local Standard of Rest velocity. The prefix is CHVC for the clouds satisfying both of our isolation criteria; the prefix is CHVC: for clouds for which the isolation is less clear; the prefix is CHVC? for clouds which have some other shortcoming (see notes) in their isolation designation; while HVC is used to indicate clouds connected to extended complexes. The longitude, latitude, and velocity refer to the intensity-weighted averages of all pixels which are assigned to the cloud.

Columns 3 and 4: J2000 right ascension and declination coordinates, respectively, of the position listed in Col. 2 .

Columns 5, 6, and 7: Radial velocities measured with respect to the Local Standard of Rest, the Galactic Standard of Rest, and the Local Group Standard of Rest systems, respectively. The Galactic Standard of Rest reference frame is defined by $V_{\mathrm{GSR}}=V_{\mathrm{LSR}}+220 \cos (b) \sin (l)$; the Local Group Standard of Rest frame, by $V_{\mathrm{LGSR}}=V_{\mathrm{GSR}}-$ $62 \cos (b) \cos (l)+40 \cos (b) \sin (l)-35 \sin (b)$. The input values of $l, b$, and $V_{\mathrm{LSR}}$ are those listed in Col. 2 .

Column 8: Velocity FWHM of the spectrum which passes through the peak-intensity pixel of the cloud.

Columns 9, 10, and 11: Angular FWHM major axis, minor axis, and major axis position angle, respectively. Using the appropriate velocity-integrated moment-map image, an ellipse was fit to the contour with half the value of the maximum column density of the cloud (Col. 13). The position angle is positive in the direction of increasing Galactic longitude, with a value of zero when the cloud is aligned pointing toward the Galactic north pole.

Column 12: Peak brightness temperature of the cloud.

Column 13: Maximum column density of the cloud in units of $10^{20} \mathrm{~cm}^{-2}$.

Column 14: Total flux of the cloud, in units of $\mathrm{Jy} \mathrm{km} \mathrm{s}^{-1}$.

Column 15: Indications of occurances of the features in other catalogs of anomalous-velocity clouds, coded as follows, with the number in the appropriate catalog indicated in the table: WW, for the Wakker \& van Woerden (1991) analysis of the Hulsbosch \& Wakker (1988) and Bajaja (1985) surveys; BB, for the BB99 visual search of the LDS; HP, for the Putman et al. (2002) application of the algorithm described here to the HIPASS data; and WSRT, for confirming observations in this study.

Column 16: References to earlier studies of individual features, and some explanatory notes. The references to earlier studies of the tabulated features are coded as follows: BBOQ, Braun \& Burton (2000); BKP01, Brüns et al. (2001); BBCQ1, Burton et al. (2001); CM79, Cohen \& Mirabel (1979); D75, Davies (1975); G81, Giovanelli (1981); HSP01, Hoffmann et al. (2002); H78, Hulsbosch (1978); H92, Henning (1992); M81, Mirabel (1981); MC79, Mirabel \& Cohen (1979); SS74, Saraber \& Shane (1974); and W79, Wright (1979). The notes carry the following meanings: (1) denotes objects with $V_{\mathrm{DEV}}<70 \mathrm{~km} \mathrm{~s}^{-1}$ but which are nevertheless entered in the catalog, for reasons explained in the following subsection; (2) denotes objects at $\delta<-28^{\circ}$ but which are nevertheless entered in the catalog, as explained in the following subsection; (3) denotes the object CHVC? 110.6-07.0 - 466 which appears incompletely on the low-velocity wing of the LDS profile but which is amply known from earlier work and therefore is entered in the catalog; (4) denotes an object with a $T_{\text {peak }}$ which falls just below the $5 \sigma$ level of the LDS but which has been amply confirmed in the WSRT imaging of Braun \& Burton (2000); and (5) denotes an object which has an enhanced background level in excess of the nominal $1.5 \times 10^{18} \mathrm{~cm}^{-2}$ $N_{\mathrm{HI}}$ cutoff, but for which the background is sufficiently uniform to suggest that it might be unrelated to the source in question.

Each of the individual isolated objects retrieved from the LDS by the search algorithm and listed in Table 2 is illustrated in Figs. 5-9 by an image of integrated $\mathrm{H}_{\mathrm{I}}$ emission, paired with a spectrum. The images show $\mathrm{H}_{\mathrm{I}}$ integrated over the velocity range of emission which is considered part of the cloud; the gray-scale intensities are given by the color bar in units of $\mathrm{K} \mathrm{km} \mathrm{s}^{-1}$. The associated spectrum refers to the direction in the $0.5 \times 0.5$ grid nearest to the peak of the integrated emission. Inspection of the Digital Sky Survey in the region of each of these images did not show a clear optical counterpart for any of the listed objects.

The arrangement on the sky of the isolated objects listed in Table 2 is shown superposed on velocity-integrated sky images in Fig. 10, for the velocity range $-450<V_{\mathrm{LSR}}<-150 \mathrm{~km} \mathrm{~s}^{-1}$; in Fig. 11, for the range $-150<V_{\mathrm{LSR}}<-90 \mathrm{~km} \mathrm{~s}^{-1}$; and in Fig. 12, for the range $+90<V_{\mathrm{LSR}}<+150 \mathrm{~km} \mathrm{~s}^{-1}$. We briefly comment on the sky deployment below; de Heij et al. (2002) discuss it more fully in conjunction with the southernhemisphere catalog.

The distribution of isolated object sizes and linewidths are shown in Fig. 13. Although the only limit on angular size we have imposed is the $10^{\circ} \times 10^{\circ}$ dimension of our initial column density image of each candidate, the distribution is strongly peaked with a median at $1^{\circ} \mathrm{FWHM}$ and does not extend beyond 2 .2. Sharply bounded anomalous-velocity objects apparently can be well described with the term: Compact High Velocity Clouds. The distribution of velocity $F W H M$ is also strongly peaked at about $25 \mathrm{~km} \mathrm{~s}^{-1}$, although the distribution does extend out to $100 \mathrm{~km} \mathrm{~s}^{-1}$. 

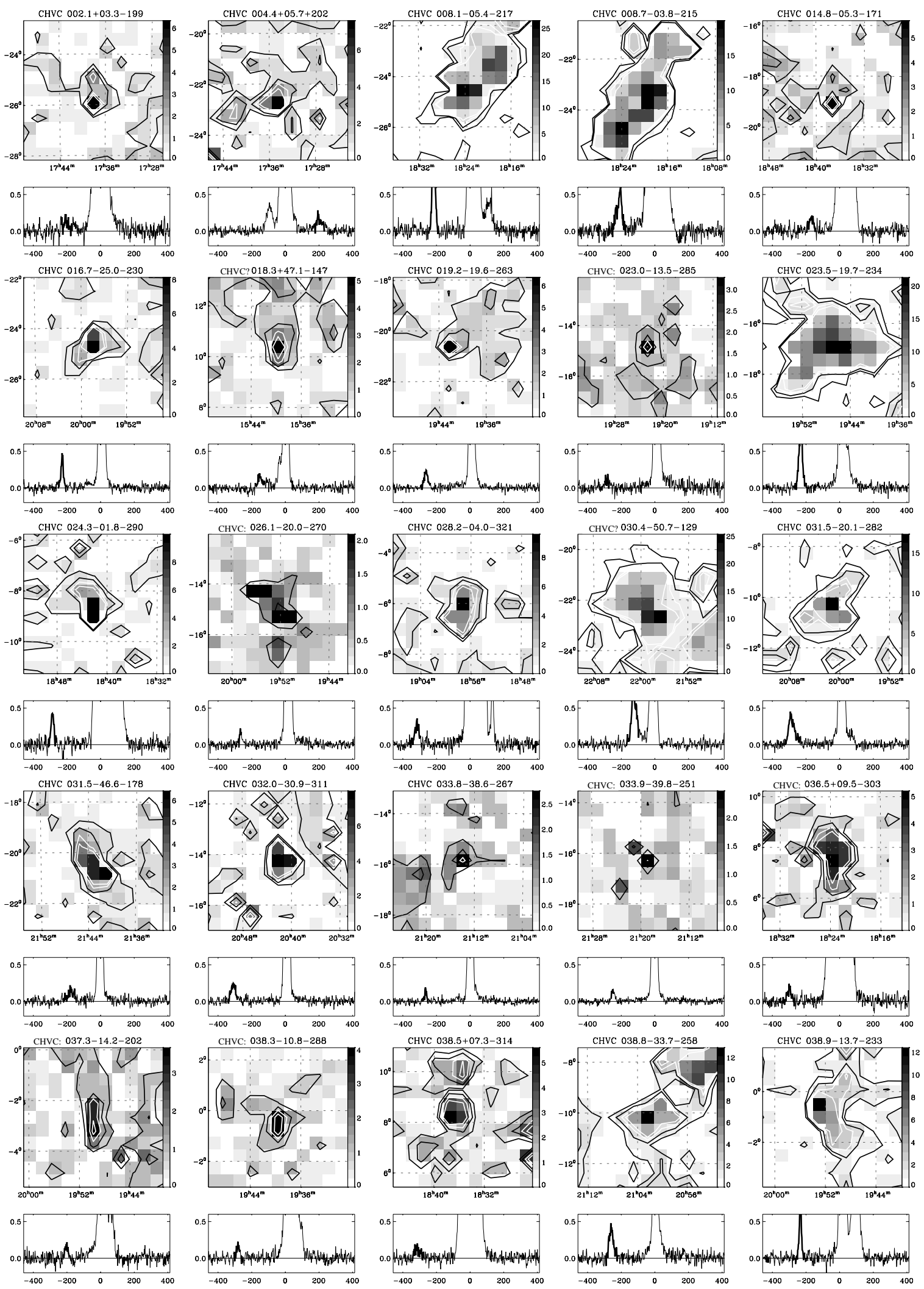

Fig. 5. Images of integrated $\mathrm{H}_{\mathrm{I}}$ emission, paired with a representative spectrum for all of the fully and partially isolated objects (CHVCs, CHVC:s and CHVC?s) retrieved from the LDS by the search algorithm, confirmed in independent data, and cataloged in Table 2. The images show $\mathrm{H}_{\mathrm{I}}$ integrated over the entire velocity range of emission which is considered part of the cloud; the gray-scale intensities are given by the color bar in units of $\mathrm{K} \mathrm{km} \mathrm{s}^{-1}$. Contours are drawn for $N_{\mathrm{HI}}=1.5,3,4.5$ and $6 \times 10^{18} \mathrm{~cm}^{-2}$. The associated spectrum refers to the direction in the $0.5^{\circ} \times 0.5^{\circ}$ grid nearest to the peak of the integrated emission. The data for the images and for the spectra were extracted from the LDS, not from the independent confirming material. 

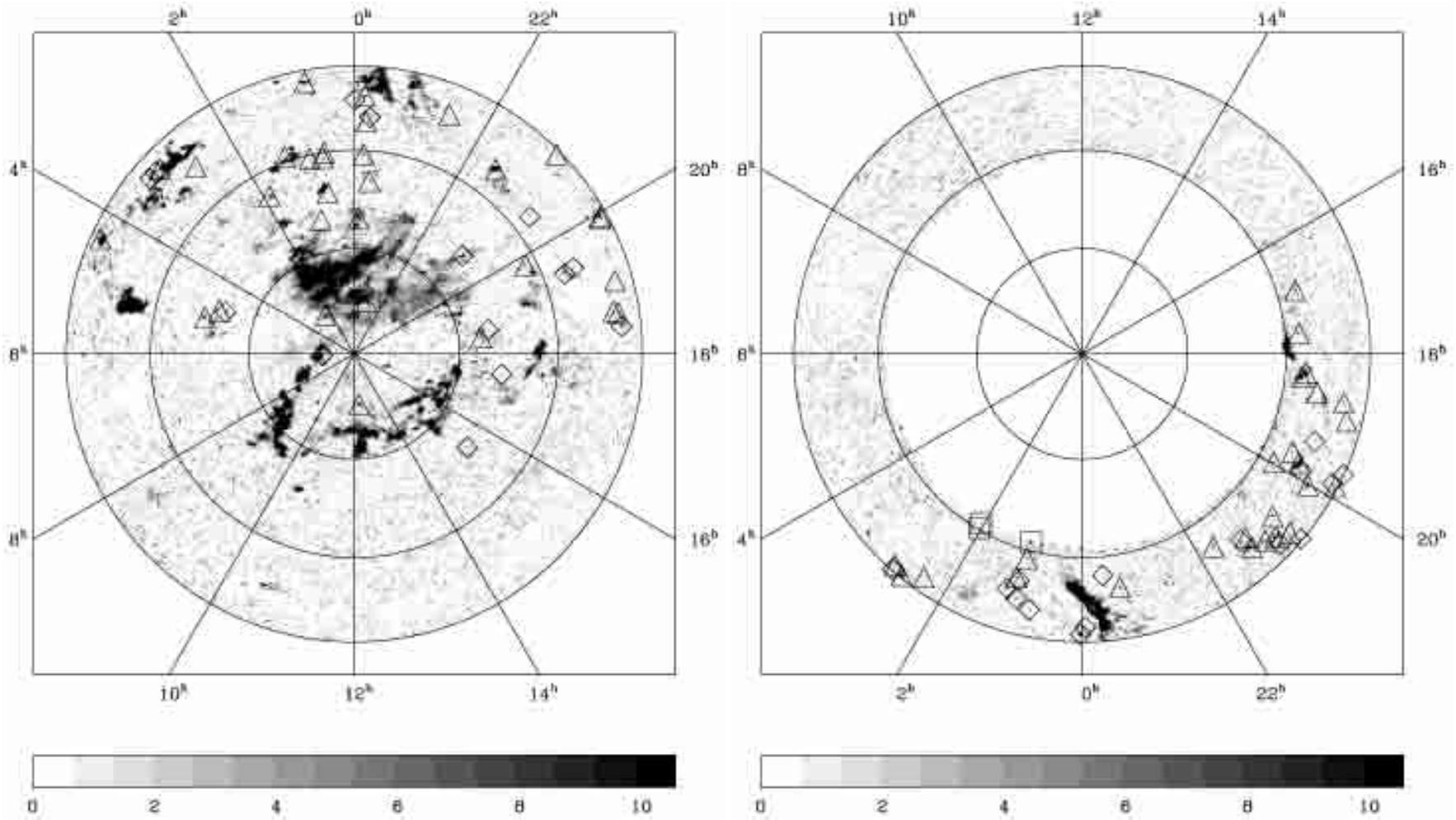

Fig. 10. Distribution of CHVCs found in the velocity range $-450<V_{\mathrm{LSR}}<-150 \mathrm{~km} \mathrm{~s}^{-1}$ across the northern hemisphere (left) and the portion of the southern hemisphere at $\delta>-30^{\circ}$ accessed by the LDS (right). Open diamonds indicate the locations of individual CHVCs. The gray shadings indicate the total emission in this velocity range, with the color bar giving the scale in units of $\mathrm{K} \mathrm{km} \mathrm{s}^{-1}$. Circles of constant declination are draw for $\delta=0^{\circ}, \pm 30^{\circ}$, and $\pm 60^{\circ}$. The scale-bar shows units of $\mathrm{K} \mathrm{km} \mathrm{s}^{-1}$. Bright, isolated clouds in the images which are not centered within a CHVC symbol are nearby galaxies. Due to the large range of integration, some individual compact clouds - of narrow width or otherwise of low total flux, do not clearly show up. Confirmation has proven, however, that they are real.
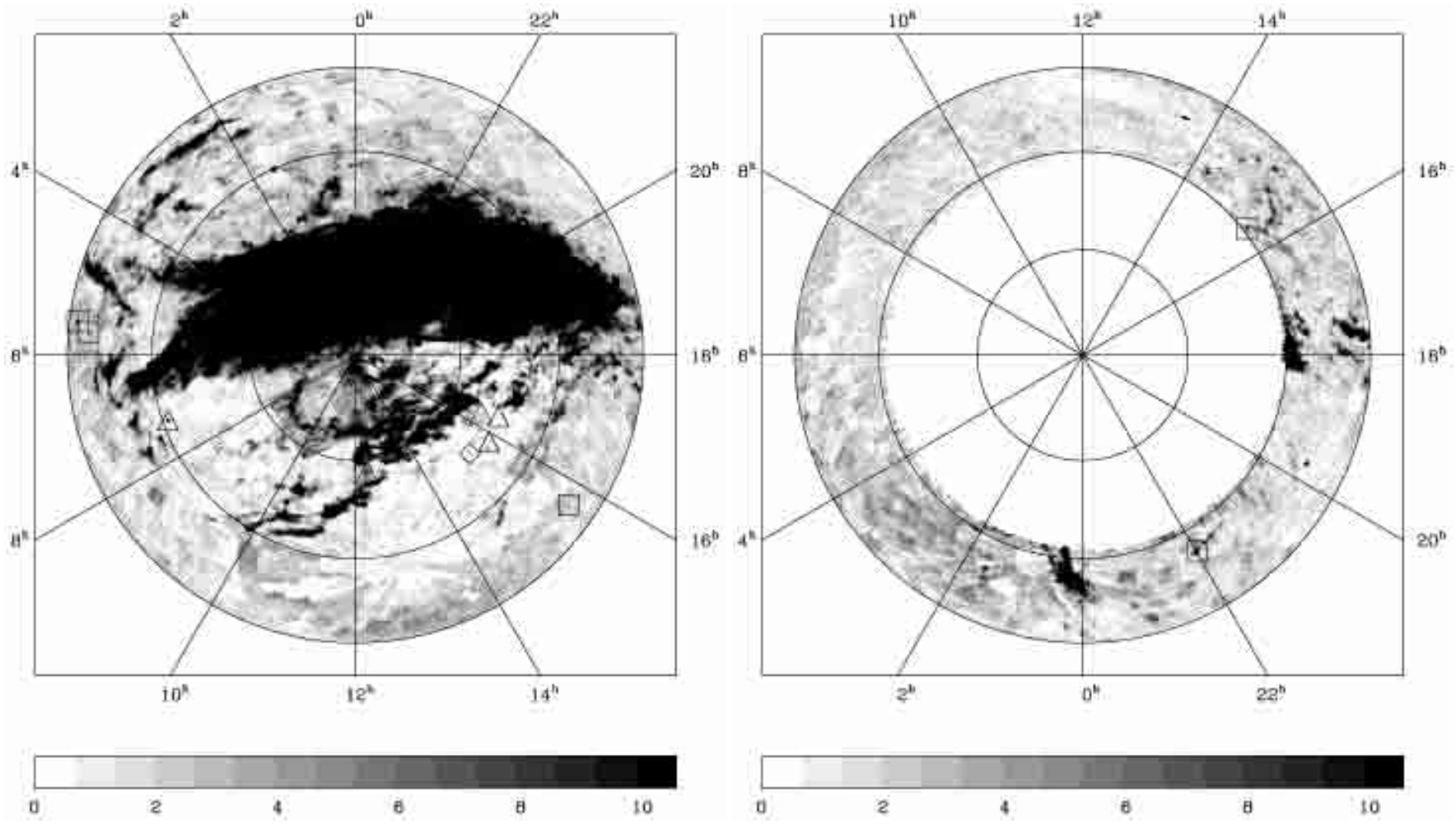

Fig. 11. Like Fig. 10, but with the velocity integration ranging from $V_{\mathrm{LSR}}=-150 \mathrm{~km} \mathrm{~s}^{-1}$ to $V_{\mathrm{LSR}}=-90 \mathrm{~km} \mathrm{~s}^{-1}$. 
Table 1. Catalog of High-Velocity Clouds identified in the Leiden/Dwingeloo Survey.

Table 2. Isolated, high-velocity clouds identified in the Leiden/Dwingeloo Survey.

\begin{tabular}{|c|c|c|c|c|c|c|c|c|c|c|c|c|c|c|c|}
\hline$\#$ & $\begin{array}{c}\text { designation } \\
l l l . l \pm b b . b \pm V V V \\
(2)\end{array}$ & $\begin{array}{c}\mathrm{RA} \\
\mathrm{h} \mathrm{m} \\
(3) \\
\end{array}$ & $\begin{array}{c}\text { DEC } \\
\circ, \\
(4) \\
\end{array}$ & $\begin{array}{c}V_{\mathrm{LSR}} \\
\mathrm{km} \mathrm{s}^{-1} \\
(5) \\
\end{array}$ & $\begin{array}{c}V_{\mathrm{GSR}} \\
\mathrm{km} \mathrm{s}^{-1} \\
(6) \\
\end{array}$ & $\begin{array}{c}V_{\mathrm{LGSR}} \\
\mathrm{km} \mathrm{s}^{-1} \\
(7) \\
\end{array}$ & $\begin{array}{c}F W H M \\
\mathrm{~km} \mathrm{~s}^{-1} \\
(8) \\
\end{array}$ & $\begin{array}{c}\text { MAJ } \\
\circ \\
(9) \\
\end{array}$ & $\begin{array}{c}\text { MIN } \\
\circ \\
(10) \\
\end{array}$ & $\begin{array}{c}\mathrm{PA} \\
\circ \\
(11) \\
\end{array}$ & $\begin{array}{c}T_{\text {peak }} \\
\mathrm{K} \\
(12) \\
\end{array}$ & $\begin{array}{c}N_{\mathrm{HI}} \\
10^{20} \mathrm{~cm}^{-2} \\
(13)\end{array}$ & $\begin{array}{c}\text { FLUX } \\
\mathrm{Jy} \mathrm{km} \mathrm{s}^{-1} \\
(14)\end{array}$ & $\begin{array}{l}\text { catalog } \\
\text { numbers } \\
(15)\end{array}$ & $\begin{array}{l}\text { references } \\
\text { and notes } \\
(16)\end{array}$ \\
\hline 1 & "CHVC 002.1+03.3-199 & 1738.1 & $\overline{-2526}$ & $\overline{-199}$ & $\overline{-191}$ & $\overline{-253}$ & 38 & 0.8 & $\overline{0.8}$ & 0 & 0.31 & $\overline{0.15}$ & 112 & HP19 & \\
\hline 2 & CHVC 004.4+05.7+202 & 1734.5 & -2215 & 202 & 219 & 157 & 32 & 0.9 & 0.6 & 170 & 0.28 & 0.16 & 121 & HP45 & \\
\hline 3 & CHVC 008.1-05.4-217 & 1824.4 & -2434 & -217 & -186 & -238 & 20 & 0.8 & 0.8 & 0 & 1.26 & 0.51 & 1139 & WW307, HP90 & SS74 \\
\hline 4 & CHVC 008.7-03.8-215 & 1819.5 & -2315 & -215 & -182 & -235 & 37 & 2.6 & 0.7 & 180 & 0.61 & 0.38 & 736 & HP92 & SS74 \\
\hline 5 & CHVC 014.8-05.3-171 & 1837.3 & -1834 & -171 & -115 & -161 & 58 & 0.4 & 0.4 & 0 & 0.21 & 0.11 & 41 & HP153 & \\
\hline 6 & CHVC 016.7-25.0-230 & 1957.9 & -2445 & -230 & -173 & -202 & 14 & 0.8 & 0.8 & 0 & 0.47 & 0.15 & 88 & BB1 & M81 \\
\hline 7 & CHVC? 018.3+47.1-147 & 1539.7 & +1023 & -147 & -100 & -157 & 53 & 0.8 & 0.8 & 0 & 0.23 & 0.12 & 97 & WW57, BB2 & B00; (5) \\
\hline 8 & CHVC 019.2-19.6-263 & 1940.0 & -2039 & -263 & -195 & -226 & 23 & 0.4 & 0.4 & 0 & 0.24 & 0.12 & 49 & HP192 & \\
\hline 9 & CHVC: $023.0-13.5-285$ & 1922.6 & -1452 & -285 & -202 & -234 & 29 & 0.4 & 0.4 & 0 & 0.19 & 0.07 & 35 & HP221 & \\
\hline 10 & CHVC 023.5-19.7-234 & 1947.0 & -1657 & -234 & -152 & -179 & 23 & 2.3 & 1.4 & 140 & 0.87 & 0.41 & 1209 & WW385, HP223 & \\
\hline 11 & CHVC 024.3-01.8-290 & 1842.2 & -0831 & -290 & -199 & -238 & 20 & 0.8 & 0.8 & 0 & 0.43 & 0.18 & 148 & WW302, BB3, HP234 & \\
\hline 12 & CHVC: $026.1-20.0-270$ & 1952.4 & -1448 & -270 & -179 & -203 & 14 & 0.8 & 0.8 & 0 & 0.22 & 0.07 & 36 & WW385, HP246 & \\
\hline 13 & CHVC 028.2-04.0-321 & 1857.3 & -0601 & -321 & -217 & -250 & 27 & 0.4 & 0.4 & 0 & 0.36 & 0.18 & 107 & HP268 & \\
\hline 14 & CHVC? 030.4-50.7-129 & 2158.9 & -2210 & -129 & -59 & -53 & 36 & 0.8 & 0.8 & 0 & 0.73 & 0.53 & 1484 & BB4, HP283 & (5) \\
\hline 15 & CHVC 031.5-20.1-282 & 2001.2 & -1016 & -282 & -175 & -193 & 38 & 0.4 & 0.4 & 0 & 0.45 & 0.32 & 215 & WW386, BB5, HP294 & \\
\hline 16 & CHVC $031.5-46.6-178$ & 2143.2 & -2019 & -178 & -99 & -95 & 29 & 0.8 & 0.8 & 0 & 0.27 & 0.17 & 134 & HP291 & \\
\hline 17 & CHVC 032.0-30.9-311 & 2042.2 & -1415 & -311 & -211 & -220 & 36 & 0.8 & 0.8 & 0 & 0.25 & 0.15 & 80 & WW443, BB6, HP299 & \\
\hline 18 & CHVC 033.8-38.6-267 & 2113.9 & -1552 & -267 & -172 & -173 & 10 & 0.4 & 0.4 & 0 & 0.26 & 0.06 & 13 & WW489, HP326 & \\
\hline 19 & CHVC: $033.9-39.8-251$ & 2118.9 & -1618 & -251 & -157 & -157 & 16 & 0.4 & 0.4 & 0 & 0.13 & 0.04 & 12 & HP322 & \\
\hline 20 & CHVC: $036.5+09.5-303$ & 1823.7 & +0729 & -303 & -174 & -206 & 62 & 2.2 & 1.3 & -80 & 0.24 & 0.10 & 134 & WW254 & \\
\hline 21 & CHVC: $037.3-14.2-202$ & 1949.8 & -0240 & -202 & -73 & -88 & 38 & 0.9 & 0.6 & 10 & 0.23 & 0.09 & 62 & WW345, HP345 & \\
\hline 22 & CHVC: $038.3-10.8-288$ & 1939.6 & -0016 & -288 & -155 & -171 & 39 & 0.8 & 0.8 & 0 & 0.22 & 0.10 & 76 & WW345, HP356 & \\
\hline 23 & CHVC $038.5+07.3-314$ & 1835.4 & +0812 & -314 & -178 & -206 & 41 & 0.8 & 0.8 & 0 & 0.20 & 0.11 & 70 & WW268 & \\
\hline 24 & CHVC 038.8-33.7-258 & 2102.4 & -1011 & -258 & -144 & -144 & 29 & 0.8 & 0.8 & 0 & 0.49 & 0.25 & 210 & WW460, BB8, HP361 & \\
\hline 25 & CHVC 038.9-13.7-233 & 1950.9 & -0101 & -233 & -99 & -113 & 14 & 0.8 & 0.8 & 0 & 0.79 & 0.24 & 235 & WW345, HP365 & \\
\hline 26 & CHVC 039.0-37.1-239 & 2115.1 & -1132 & -239 & -129 & -126 & 25 & 0.8 & 0.8 & 0 & 0.34 & 0.15 & 128 & WW482, BB7, HP360 & \\
\hline 27 & CHVC 039.6-31.0-272 & 2053.9 & -0823 & -272 & -151 & -152 & 25 & 1.8 & 1.5 & -80 & 0.37 & 0.21 & 326 & WW442, BB9, HP370 & \\
\hline 28 & CHVC $040.0+07.6-314$ & 1837.1 & +0944 & -314 & -173 & -200 & 19 & 0.8 & 0.8 & 0 & 0.20 & 0.06 & 23 & WW268 & HW88 \\
\hline 29 & CHVC $040.2+00.5-279$ & 1903.0 & +0644 & -279 & -137 & -159 & 42 & 0.8 & 0.8 & 0 & 0.19 & 0.11 & 61 & WW289, BB10 & \\
\hline 30 & CHVC: $040.4-73.4-169$ & 2339.1 & -2349 & -169 & -128 & -101 & 20 & 0.8 & 0.8 & 0 & 0.14 & 0.06 & 46 & HP367 & \\
\hline 31 & CHVC 041.1-27.3-239 & 2043.1 & -0533 & -239 & -111 & -113 & 26 & 0.8 & 0.8 & 0 & 0.25 & 0.11 & 59 & WW419, HP379 & \\
\hline 32 & CHVC 042.9-12.9-265 & 1955.6 & +0243 & -265 & -119 & -129 & 51 & 0.8 & 0.8 & 0 & 0.32 & 0.16 & 95 & WW348, B11, HP384 & G81 \\
\hline 33 & CHVC 042.9-13.3-315 & 1956.8 & +0238 & -315 & -169 & -179 & 27 & 1.2 & 1.0 & -60 & 0.64 & 0.40 & 396 & WW348, B11, HP384 & G81 \\
\hline 34 & CHVC: $043.0-29.9-217$ & 2055.3 & -0518 & -217 & -87 & -85 & 25 & 0.8 & 0.8 & 0 & 0.34 & 0.22 & 262 & WW445, HP389 & \\
\hline 35 & CHVC: $045.5-24.5-228$ & 2040.9 & -0047 & -228 & -85 & -84 & 36 & 0.8 & 0.8 & 0 & 0.26 & 0.13 & 84 & WW409, HP404 & \\
\hline 36 & CHVC $050.0-68.2-193$ & 2323.3 & -1903 & -193 & -131 & -102 & 30 & 0.8 & 0.8 & 0 & 0.24 & 0.13 & 99 & BB13, HP421 & \\
\hline 37 & CHVC 050.2-27.1-274 & 2058.5 & +0134 & -274 & -124 & -116 & 26 & 0.9 & 0.6 & 0 & 0.19 & 0.10 & 42 & WW421, HP420 & \\
\hline 38 & CHVC: $054.1+01.5-197$ & 1925.9 & +1929 & -197 & -19 & -24 & 17 & 0.4 & 0.4 & 0 & 0.30 & 0.08 & 30 & WW283 & \\
\hline 39 & CHVC: $057.0+03.7-209$ & 1923.5 & +2259 & -209 & -25 & -28 & 24 & 3.8 & 0.9 & 70 & 0.32 & 0.11 & 132 & WW274 & \\
\hline 40 & CHVC: $065.9-09.4-273$ & 2032.2 & +2348 & -273 & -75 & -58 & 29 & 0.9 & 0.6 & 170 & 0.22 & 0.11 & 74 & WW327 & \\
\hline 41 & CHVC $069.0+03.8-236$ & 1949.4 & +3333 & -236 & -31 & -19 & 26 & 0.8 & 0.8 & 0 & 0.31 & 0.15 & 114 & WW273, BB15 & $\mathrm{BB} 00$ \\
\hline 42 & CHVC $070.3+50.9-144$ & 1548.0 & +4358 & -144 & -13 & -30 & 16 & 1.7 & 1.1 & -50 & 0.31 & 0.12 & 84 & WW44, BB16 & \\
\hline 43 & CHVC $070.6+43.8-142$ & 1627.4 & +4507 & -142 & 8 & -4 & 22 & 0.8 & 0.8 & 0 & 0.19 & 0.06 & 30 & WW72 & \\
\hline 44 & CHVC $072.0-21.9-333$ & 2130.2 & +2033 & -333 & -139 & -108 & 24 & 2.0 & 1.2 & -60 & 0.75 & 0.40 & 674 & WW394 & \\
\hline 45 & CHVC: $073.4+33.3-206$ & 1728.2 & +4711 & -206 & -30 & -32 & 26 & 1.2 & 1.2 & 0 & 0.25 & 0.07 & 67 & Ww90 & \\
\hline 46 & CHVC: $076.9+55.5-115$ & 1516.9 & +4632 & -115 & 6 & -9 & 24 & 1.3 & 0.9 & 70 & 0.25 & 0.10 & 102 & WW28 & \\
\hline 47 & CHVC $077.5-38.9-320$ & 2233.5 & +1132 & -320 & -152 & -110 & 20 & 1.3 & 0.8 & 40 & 0.25 & 0.10 & 52 & WW485 & \\
\hline 48 & CHVC $078.1+44.1-149$ & 1621.8 & +5023 & -149 & 5 & 0 & 29 & 0.8 & 0.8 & 0 & 0.19 & 0.10 & 60 & WW70 & \\
\hline 49 & CHVC: $078.4+54.2-158$ & 1521.8 & +4749 & -158 & -32 & -45 & 27 & 1.5 & 1.1 & 0 & 0.19 & 0.08 & 88 & WW28 & \\
\hline 50 & CHVC: $080.1+22.3-209$ & 1841.4 & +5059 & -209 & -8 & 5 & 20 & 1.3 & 0.9 & 70 & 0.23 & 0.07 & 65 & WW191 & \\
\hline 51 & CHVC $082.2+24.6-196$ & 1829.8 & +5325 & -196 & 2 & 16 & 20 & 1.3 & 1.3 & -90 & 0.35 & 0.13 & 300 & WW182 & \\
\hline 52 & CHVC: $087.2+02.7-296$ & 2048.7 & +4801 & -296 & -76 & -41 & 45 & 0.8 & 0.8 & 0 & 0.24 & 0.13 & 116 & WW278, BB17 & \\
\hline 53 & CHVC: $089.4-65.1-315$ & 2357.3 & -0546 & -315 & -222 & -174 & 24 & 2.0 & 1.2 & 30 & 0.18 & 0.08 & 73 & WW547, HP495 & \\
\hline 54 & CHVC: $094.4-63.0-321$ & 0001.3 & -0258 & -321 & -221 & -170 & 21 & 0.8 & 0.8 & 0 & 0.36 & 0.16 & 143 & WW542, BB20, HP505 & \\
\hline 55 & CHVC 099.9-48.8-392 & 2350.5 & +1121 & -392 & -249 & -190 & 36 & 0.8 & 0.8 & 0 & 0.27 & 0.15 & 115 & WW493, BB21 & $\mathrm{BBC} 01$ \\
\hline 56 & CHVC: $101.7-41.3-430$ & 2344.8 & +1848 & -430 & -269 & -207 & 45 & 0.8 & 0.8 & 0 & 0.17 & 0.08 & 36 & WW491 & \\
\hline 57 & CHVC 103.4-40.1-414 & 2348.0 & +2022 & -414 & -250 & -187 & 24 & 1.9 & 0.9 & 40 & 0.30 & 0.14 & 155 & WW491 & HSP01 \\
\hline 58 & CHVC: $103.8-48.1-167$ & 2359.5 & +1249 & -167 & -24 & 38 & 25 & 0.4 & 0.4 & 0 & 0.21 & 0.08 & 34 & WW518 & \\
\hline
\end{tabular}

\subsection{Differences between this catalog and that of BB99}

The BB99 compilation was based on the same data as were used in the preparation of Tables 1 and 2, but on selection criteria which were somewhat different than those of the algorithm described here, and so there are several differences between the Table 2 catalog and that of BB99. The Table 2 catalog includes objects not found in the BB99 list, because it was extended to lower flux levels since new confirming data were planned; conversely, a number of objects listed by BB99 were not included in the current work. The differences are explained as follows:

BB19, BB45, BB47, BB49, BB50, BB53, BB59 and BB60:

These objects all have a $V_{\mathrm{DEV}}$ less than the $70 \mathrm{~km} \mathrm{~s}^{-1}$ limit conservatively imposed for our search. The first of these objects has been identified as a nearby LSB galaxy, Cep I, by Burton et al. (1999) and is not included in Table 2. 
Table 2. continued.

\begin{tabular}{|c|c|c|c|c|c|c|c|c|c|c|c|c|c|c|c|}
\hline$\#$ & $\begin{array}{c}\text { designation } \\
l l l . l \pm b b . b \pm V V V \\
(2) \\
\end{array}$ & $\begin{array}{c}\mathrm{RA} \\
\mathrm{h} \mathrm{m} \\
(3) \\
\end{array}$ & $\begin{array}{c}\text { DEC } \\
\circ, \\
(4) \\
\end{array}$ & $\begin{array}{c}V_{\mathrm{LSR}} \\
\mathrm{km} \mathrm{s}^{-1} \\
(5) \\
\end{array}$ & $\begin{array}{c}V_{\mathrm{GSR}} \\
\mathrm{km} \mathrm{s}^{-1} \\
(6)\end{array}$ & $\begin{array}{c}V_{\text {LGSR }} \\
\mathrm{km} \mathrm{s}^{-1} \\
(7)\end{array}$ & $\begin{array}{c}F W H M \\
\mathrm{~km} \mathrm{~s}^{-1} \\
(8) \\
\end{array}$ & $\begin{array}{c}\text { MAJ } \\
\circ \\
(9) \\
\end{array}$ & 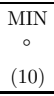 & $\begin{array}{c}\mathrm{PA} \\
\circ \\
(11) \\
\end{array}$ & $\begin{array}{c}T_{\text {peak }} \\
\mathrm{K} \\
(12) \\
\end{array}$ & $\begin{array}{c}N_{\mathrm{HI}} \\
10^{20} \mathrm{~cm}^{-2} \\
(13) \\
\end{array}$ & $\begin{array}{c}\text { FLUX } \\
\mathrm{Jy} \mathrm{km} \mathrm{s}^{-1} \\
(14) \\
\end{array}$ & $\begin{array}{l}\text { catalog } \\
\text { numbers } \\
(15) \\
\end{array}$ & $\begin{array}{l}\text { references } \\
\text { and notes } \\
(16)\end{array}$ \\
\hline 59 & CHVC $107.7-29.7-429$ & 2349.3 & +3119 & -429 & -247 & -180 & 40 & 0.4 & 0.4 & 0 & 0.29 & 0.13 & 89 & WW437, BB22 & H92 \\
\hline 60 & CHVC $108.3-21.2-402$ & 2340.2 & +3940 & -402 & -208 & -141 & 33 & 0.8 & 0.8 & 0 & 0.22 & 0.08 & 52 & BB23, WW389 & \\
\hline 61 & CHVC? $110.6-07.0-466$ & 2327.1 & +5350 & -466 & -262 & -199 & 25 & 1.0 & 0.9 & 70 & 0.17 & 0.08 & 91 & WW318, BB24 & H78, CM79, WS91; (3) \\
\hline 62 & CHVC $113.7-10.6-442$ & 2353.0 & +5113 & -442 & -244 & -177 & 11 & 0.9 & 0.6 & 0 & 0.77 & 0.18 & 101 & WW330, BB25 & H78, CM79, WS91 \\
\hline 63 & CHVC? $115.4+13.4-260$ & 2256.9 & +7433 & -260 & -67 & -14 & 95 & 1.3 & 0.8 & -80 & 0.16 & 0.30 & 375 & BB26 & BBO0; (4); (5) \\
\hline 64 & CHVC $118.2-58.1-373$ & 0041.5 & +0439 & -373 & -270 & -207 & 31 & 0.8 & 0.8 & 0 & 0.71 & 0.40 & 536 & WW532, BB27 & MC79, G81 \\
\hline 65 & CHVC $118.5-32.6-386$ & 0034.1 & +3005 & -386 & -223 & -149 & 26 & 0.4 & 0.4 & 0 & 0.15 & 0.07 & 19 & WSRT & \\
\hline 66 & CHVC: $119.0-73.1-300$ & 0046.7 & -1014 & -300 & -244 & -191 & 34 & 0.4 & 0.4 & 0 & 0.15 & 0.09 & 19 & WW555, BB28, HP520 & \\
\hline 67 & CHVC 119.2-31.1-384 & 0036.2 & +3139 & -384 & -220 & -146 & 19 & 0.8 & 0.8 & 0 & 0.33 & 0.12 & 117 & WW444, BB29 & W79 \\
\hline 68 & CHVC $120.2-20.0-441$ & 0037.4 & +4247 & -441 & -262 & -188 & 18 & 0.4 & 0.4 & -80 & 0.29 & 0.10 & 22 & WSRT & D75 \\
\hline 69 & CHVC $122.9-31.8-325$ & 0051.4 & +3104 & -325 & -168 & -93 & 34 & 0.4 & 0.4 & 0 & 0.20 & 0.11 & 51 & WW446, BB30 & \\
\hline 70 & CHVC $123.7-12.4-214$ & 0055.8 & +5026 & -214 & -36 & 38 & 31 & 1.2 & 1.0 & -60 & 0.28 & 0.13 & 125 & WW287 & \\
\hline 71 & CHVC $125.3+41.3-205$ & 1222.5 & +7543 & -205 & -70 & -42 & 7 & 0.8 & 0.8 & 0 & 1.99 & 0.31 & 252 & WW84, BB31 & $\mathrm{BB} 00, \mathrm{BKP} 01$ \\
\hline 72 & CHVC $128.6+14.7-306$ & 0228.5 & +7630 & -306 & -140 & -81 & 12 & 0.8 & 0.8 & 0 & 0.50 & 0.15 & 117 & WW231, BB32 & \\
\hline 73 & CHVC $130.0-34.2-367$ & 0118.0 & +2820 & -367 & -228 & -150 & 13 & 0.8 & 0.8 & 0 & 0.42 & 0.11 & 54 & WW466 & \\
\hline 74 & CHVC $130.8+60.1-121$ & 1222.9 & +5633 & -121 & -38 & -33 & 17 & 1.5 & 1.1 & 150 & 0.29 & 0.12 & 154 & WW17 & \\
\hline 75 & CHVC: $132.0-75.8-304$ & 0100.6 & -1307 & -304 & -264 & -212 & 34 & 1.7 & 1.2 & 30 & 0.20 & 0.11 & 98 & WW557, BB33, HP530 & \\
\hline 76 & CHVC: $132.7+25.3-207$ & 0612.0 & +8101 & -207 & -61 & -11 & 11 & 1.6 & 1.3 & -90 & 0.54 & 0.09 & 199 & WW117 & \\
\hline 77 & CHVC $136.1-23.5-153$ & 0152.8 & +3749 & -153 & -13 & 68 & 24 & 1.9 & 1.2 & 40 & 0.44 & 0.24 & 526 & WW404 & \\
\hline 78 & CHVC: $141.4-81.9-223$ & 0102.2 & -1927 & -223 & -204 & -159 & 25 & 0.8 & 0.8 & 0 & 0.14 & 0.06 & 26 & HP534 & \\
\hline 79 & CHVC: $145.2-77.6-273$ & 0110.8 & -1535 & -273 & -246 & -196 & 24 & 0.8 & 0.8 & 0 & 0.23 & 0.12 & 92 & WW560, BB34, HP537 & \\
\hline 80 & CHVC 148.9-82.5-269 & 0105.5 & -2018 & -269 & -254 & -210 & 20 & 0.8 & 0.8 & 0 & 0.39 & 0.18 & 114 & BB36, HP538 & \\
\hline 81 & CHVC: $155.5+04.0-155$ & 0448.0 & +5117 & -155 & -64 & 7 & 46 & 0.8 & 0.8 & 0 & 0.64 & 0.18 & 142 & WW247 & \\
\hline 82 & CHVC $157.1+02.9-186$ & 0448.7 & +4922 & -186 & -101 & -30 & 12 & 0.9 & 0.6 & 180 & 0.36 & 0.10 & 49 & WW275, BB38 & \\
\hline 83 & CHVC $157.7-39.3-287$ & 0240.9 & +1604 & -287 & -222 & -144 & 12 & 0.4 & 0.4 & 0 & 0.22 & 0.08 & 24 & WW486, BB39 & BBC01; HSP01 \\
\hline 84 & CHVC $161.6+02.7-186$ & 0504.9 & +4543 & -186 & -117 & -47 & 23 & 0.8 & 0.8 & 0 & 0.64 & 0.32 & 219 & WW277, BB40 & \\
\hline 85 & CHVC $170.8-42.3-217$ & 0305.8 & +0745 & -217 & -191 & -117 & 24 & 0.8 & 0.8 & 0 & 0.43 & 0.15 & 117 & WW490 & \\
\hline 86 & CHVC $171.3-53.6-238$ & 0236.6 & -0055 & -238 & -218 & -150 & 28 & 0.8 & 0.8 & 0 & 0.53 & 0.28 & 341 & WW525, BB41, HP570 & H78 \\
\hline 87 & CHVC 171.7-59.7-234 & 0221.2 & -0536 & -234 & -218 & -154 & 23 & 0.8 & 0.8 & 0 & 0.23 & 0.10 & 48 & WW536, BB43, HP571 & \\
\hline 88 & CHVC: $172.3-41.9-292$ & 0310.2 & +0717 & -292 & -270 & -197 & 42 & 0.9 & 0.6 & 160 & 0.28 & 0.11 & 69 & WW501 & \\
\hline 89 & CHVC: $173.4-51.9-230$ & 0245.3 & -0031 & -230 & -215 & -146 & 30 & 0.8 & 0.8 & 0 & 0.25 & 0.12 & 53 & WW525, HP572 & \\
\hline 90 & CHVC: $173.7-40.5-203$ & 0317.2 & +0732 & -203 & -185 & -112 & 33 & 0.4 & 0.4 & 0 & 0.22 & 0.11 & 45 & WW467 & \\
\hline 91 & CHVC $175.8-53.0-216$ & 0246.1 & -0221 & -216 & -207 & -140 & 28 & 0.8 & 0.8 & 0 & 0.20 & 0.08 & 30 & WW525, HP573 & \\
\hline 92 & CHVC $186.3+18.8-109$ & 0716.5 & +3141 & -109 & -132 & -89 & 14 & 0.9 & 0.6 & 160 & 1.10 & 0.32 & 305 & WW215, BB44 & $\mathrm{BBC} 01$ \\
\hline 93 & CHVC $190.2-30.5-168$ & 0422.6 & +0347 & -168 & -201 & -137 & 30 & 1.3 & 1.3 & -80 & 0.55 & 0.25 & 1140 & WW467 & \\
\hline 94 & CHVC? $190.9+60.4+093$ & 1036.9 & +3410 & 93 & 73 & 69 & 30 & 1.2 & 1.1 & 80 & 0.38 & 0.22 & 324 & BB45 & BB00; (1); (5) \\
\hline 95 & CHVC? 197.5-12.0-106 & 0540.2 & +0751 & -106 & -171 & -117 & 25 & 0.8 & 0.8 & 0 & 0.48 & 0.27 & 284 & WW343, BB46 & $\mathrm{BBC01;（5)}$ \\
\hline 96 & CHVC? $200.2+29.7+080$ & 0822.2 & +2320 & 75 & 9 & 30 & 29 & 0.6 & 0.6 & 0 & 0.50 & 0.28 & 118 & BB47 & (1); (5) \\
\hline 97 & CHVC $200.6+52.3+107$ & 1000.0 & +2829 & 107 & 60 & 59 & 22 & 0.8 & 0.8 & 0 & 0.37 & 0.17 & 89 & WSRT & \\
\hline 98 & CHVC? 200.7-16.0-098 & 0532.2 & +0313 & -98 & -172 & -120 & 31 & 1.3 & 0.8 & 50 & 0.58 & 0.40 & 730 & WW362, BB48 & (5) \\
\hline 99 & CHVC? $202.2+30.4+057$ & 0827.4 & +2155 & 57 & -15 & 6 & 26 & 1.9 & 1.4 & 40 & 1.12 & 0.57 & 1796 & BB49 & $\mathrm{BBC} 01 ;(1) ;(5)$ \\
\hline 100 & CHVC? $204.2+29.8+075$ & 0827.5 & +2009 & 61 & -17 & 0 & 34 & 0.8 & 0.8 & 0 & 1.19 & 0.79 & 777 & BB50 & $\mathrm{BB} 00, \mathrm{BBC} 01 ; \quad(1) ;$ \\
\hline 101 & CHVC $217.9+28.7+145$ & 0843.1 & +0842 & 145 & 26 & 31 & 7 & 0.4 & 0.4 & 0 & 0.66 & 0.09 & 56 & WW159 & \\
\hline 102 & CHVC 218.4-87.9-260 & 0100.7 & -2718 & -260 & -264 & -229 & 29 & 0.8 & 0.8 & 0 & 0.35 & 0.15 & 102 & BB51, HP615 & \\
\hline 103 & CHVC? 224.6+35.9+082 & 0919.2 & +0659 & 82 & -43 & -51 & 36 & 1.4 & 1.2 & 80 & 0.22 & 0.16 & 307 & WW115, BB53 & (1); (5) \\
\hline 104 & CHVC $224.6-08.0+188$ & 0643.6 & -1357 & 188 & 36 & 56 & 21 & 0.8 & 0.8 & 0 & 0.32 & 0.15 & 110 & WW325, BB52, HP633 & \\
\hline 105 & CHVC 225.0-41.9+176 & 0428.6 & -2615 & 176 & 61 & 96 & 60 & 0.8 & 0.8 & 0 & 0.34 & 0.20 & 253 & BB54, HP639 & \\
\hline 106 & CHVC? 226.5-33.5+101 & 0505.9 & -2514 & 101 & -32 & -1 & 27 & 0.8 & 0.8 & 0 & 0.70 & 0.40 & 507 & BB55, HP648 & (5) \\
\hline 107 & CHVC? 228.9-74.2-168 & 0202.0 & -3022 & -174 & -219 & -182 & 33 & 0.9 & 0.8 & -60 & 0.25 & 0.16 & 136 & BB56, HP691 & (2); (5) \\
\hline 108 & CHVC 229.5+60.6+151 & 1054.5 & +1551 & 151 & 69 & 43 & 23 & 0.4 & 0.4 & 0 & 0.18 & 0.08 & 28 & BB57 & $\mathrm{BB} 00, \mathrm{BBC} 01$ \\
\hline 109 & CHVC? 235.3-73.7-150 & 0202.7 & -3210 & -157 & -208 & -174 & 23 & 0.9 & 0.8 & 80 & 0.27 & 0.12 & 102 & BB58, HP735 & (2); (5) \\
\hline 110 & CHVC? 236.7+49.8+078 & 1025.4 & +0642 & 78 & -41 & -67 & 37 & 1.3 & 1.3 & 0 & 0.19 & 0.14 & 293 & WW47, BB59 & (1); (5) \\
\hline 111 & CHVC? $241.0+53.4+089$ & 1043.5 & +0641 & 89 & -26 & -57 & 60 & 1.3 & 1.0 & 50 & 0.15 & 0.18 & 281 & WW34, BB60 & (1); (5) \\
\hline 112 & CHVC: $260.1+47.8+217$ & 1102.3 & -0546 & 217 & 72 & 26 & 49 & 0.8 & 0.8 & 0 & 0.18 & 0.10 & 33 & HP974 & \\
\hline 113 & CHVC? 284.0-84.0-174 & 0100.7 & -3247 & -174 & -196 & -167 & 29 & 1.3 & 1.2 & 0 & 0.35 & 0.19 & 351 & WW561, BB64, HP1417 & (2); (5) \\
\hline 114 & CHVC? $340.1+22.5-108$ & 1529.6 & -2843 & -108 & -177 & -257 & 32 & 1.3 & 0.8 & 60 & 0.36 & 0.23 & 280 & BB65 & (2); (5) \\
\hline 115 & CHVC: $357.5+05.6+268$ & 1717.9 & -2759 & 268 & 259 & 192 & 45 & 0.4 & 0.4 & 0 & 0.24 & 0.13 & 81 & HP1966 & \\
\hline 116 & CHVC $357.5+12.4-181$ & 1653.4 & -2358 & -181 & -190 & -260 & 51 & 0.8 & 0.8 & 0 & 0.27 & 0.14 & 97 & BB66, HP1974 & \\
\hline
\end{tabular}

But the other seven objects are included in the table to allow direct comparison with the rest of the CHVC sample. The high background $N_{\mathrm{HI}}$ levels that are inevitable with such a low deviation velocity, result in none of these objects passing the more stringent isolation criterion employed here, even though this background appears smooth on angular scales of $5-10^{\circ}$ and is conceivably unrelated to the object in question. They have been given the CHVC? designation to indicate the uncertainty in classification.

$B B 12, B B 14, B B 18, B B 35, B B 37, B B 42, B B 61, B B 62$ and $B B 63$ : Although these clouds were classified as isolated in the BB99 study, they do not satisfy the more stringent critera for isolation applied here and are therefore not included in the listing. The BB99 crition of isolation was based on the $50 \%$ contour of peak $N_{\mathrm{HI}}$; here we used the $1.5 \times 10^{18} \mathrm{~cm}^{-2}$ contour.

$B B 17, B B 20, B B 24, B B 28, B B 33$, and BB34: These 6 objects are included in Table 2 , but have been classified here as CHVC:s, based on the more restrictive criteria of the current work. There is some indication for extended emission in the environment of these sources which might be associated. 

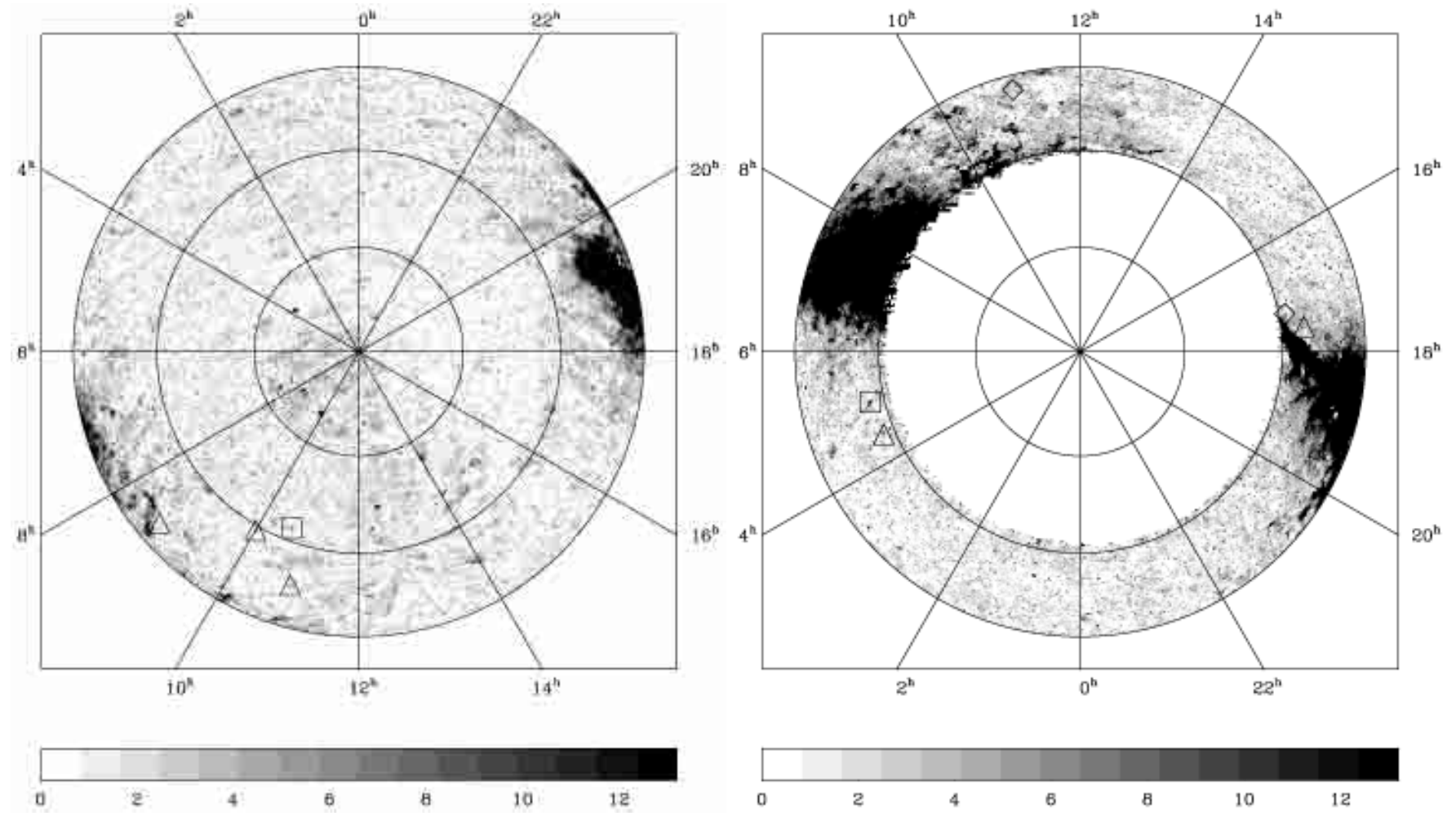

Fig. 12. Like Fig. 10, but with the velocity integration ranging from $V_{\mathrm{LSR}}=+90 \mathrm{~km} \mathrm{~s}^{-1}$ to $V_{\mathrm{LSR}}=+350 \mathrm{~km} \mathrm{~s}^{-1}$.

$B B 2, B B 4, B B 26, B B 46, B B 48, B B 55, B B 56, B B 58, B B 64$ and BB65: These 10 objects are also included in Table 2, but have been classified here as CHVC?s. In all these cases the background $N_{\mathrm{HI}}$ level exceeds our limit of $1.5 \times 10^{18} \mathrm{~cm}^{-2}$ but appears smooth on angular scales of $5-10^{\circ}$ and is conceivably unrelated to the object in question.

Thus of the 65 compact anomalous-velocity clouds listed by BB99 (excluding the nearby galaxy Cep 1), the criteria applied here have resulted in the identification of 54; of these, 31 have retained the designation $\mathrm{CHVC}$, whereas 6 have been assigned the designation CHVC: and 17 have been labeled CHVC? Some examples of reclassified objects are shown in Fig. 14. Of the total of 116 compact objects tabulated, 32 do not appear in the Wakker \& van Woerden (1991) catalog; of these 32 objects, 17 are classified in Table 2 as CHVCs.

\subsection{Differences in the zone of overlapping declinations between this catalog and the HIPASS catalog}

Putman et al. (2002) have applied the search algorithm described here to the HIPASS data, resulting in a catalog of southern compact, isolated objects. Because both the LDS catalog of CHVCs given here in Table 2 and the HIPASS catalog will be used together in an all-sky study of the kinematic and spatial properties of CHVCs, a comparison between them in the zone of overlap is interesting. The two surveys overlap in the declination range $-28^{\circ} \leq \delta \leq+2^{\circ}$, but were carried out with different observational parameters. The RMS noise figure is $10 \mathrm{mK}$ in the HIPASS material, for a channel $26 \mathrm{~km} \mathrm{~s}^{-1}$ wide and a
FWHP beam of $15^{\prime}$; the corresponding RMS value in the LDS is $70 \mathrm{mK}$, for a channel width of $1.03 \mathrm{~km} \mathrm{~s}^{-1}$ and a FWHP beam of $36^{\prime}$. After smoothing both surveys to the same spectral resolution of $26 \mathrm{~km} \mathrm{~s}^{-1}$, the $3 \sigma$ limiting column density (for emission filling each beam) is 0.47 and $0.64 \times 10^{18} \mathrm{~cm}^{-2}$ in the HIPASS and LDS respectively. Thus, while the sensitivity to well-resolved sources is comparable, the point source sensitivity of HIPASS is greater by about a factor of 3 . On the other hand, the band-pass calibration of the HIPASS data relies on reference spectra which are deemed empty of $\mathrm{H}_{\mathrm{I}}$ emission that are off-set by only a few degrees on the sky. Even with the MINMED5 method of baseline determination employed by Putman et al., there is significant filtering of extended emission, which complicates the assessment of object isolation down to a low column density limit.

The differences in point-source sensitivity on the one hand and sensitivity to very extended structures on the other, results in a larger number of faint source detections in HIPASS, but also in a different designation for some of the brighter clouds which the surveys have in common. Comparison of the results derived by applying the search algorithm to both surveys allows assessment of the robustness of the selection criteria and of the completeness of the LDS catalog.

Table 3 lists the number of anomalous-velocity features classified as either CHVCs or CHVC:s and found in the indicated peak-temperature bins (referred to the HIPASS temperature calibration scale) in both the HIPASS and LDS material in the zone of overlapping declinations. The data indicate the influence of survey sensitivity on the number of objects found. Above a peak temperature of $0.45 \mathrm{~K}$, the LDS results are as complete as those based on the HIPASS; 

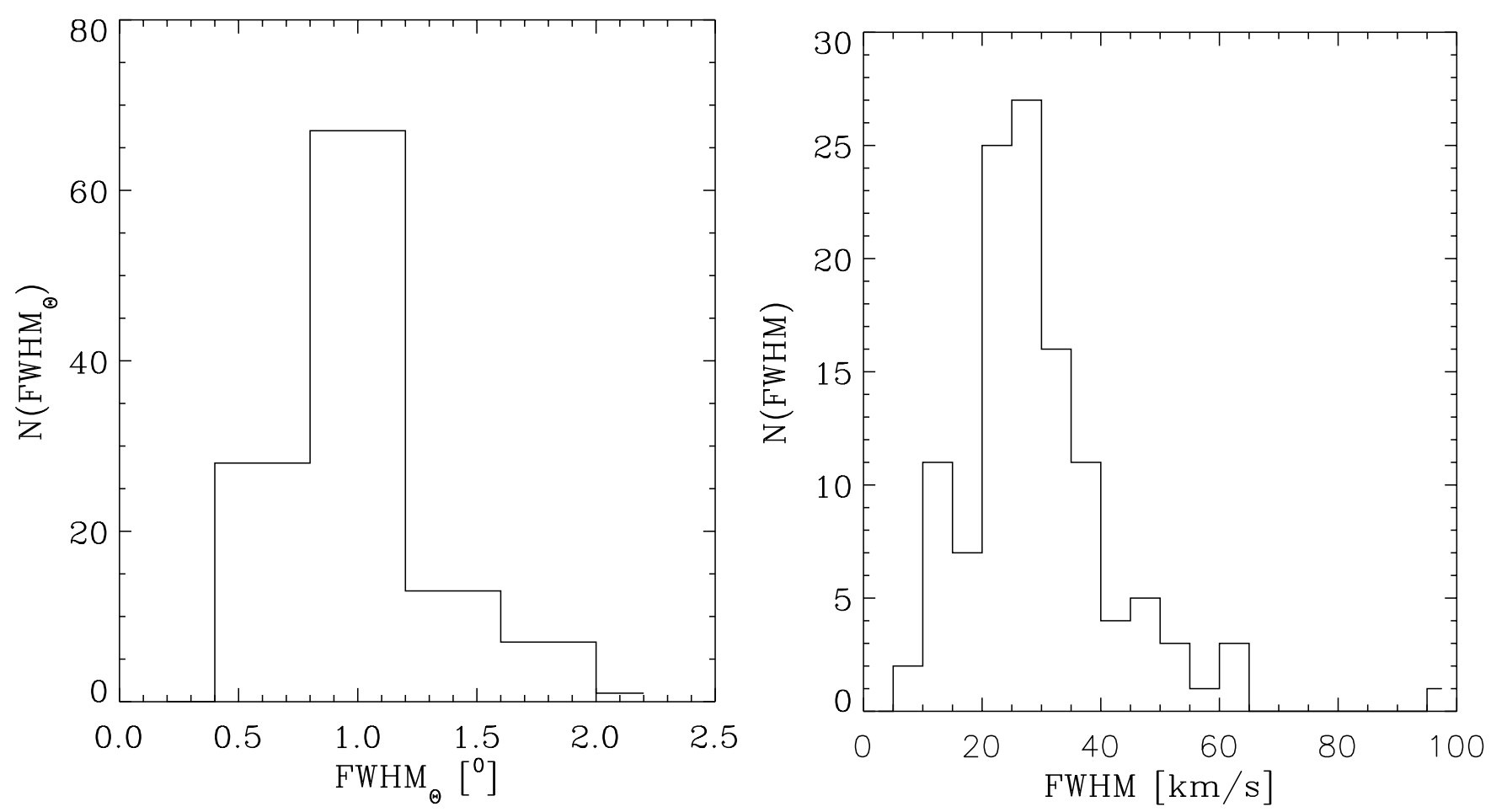

Fig. 13. Histograms of the angular size and velocity width of all of the fully and partially isolated objects (CHVCs, CHVC:s and CHVC?s) retrieved from the LDS by the search algorithm, confirmed in independent data, and cataloged in Table 2 . Although all $\mathrm{HVCs}$ less than $10^{\circ}$ in diameter where considered, the median $\mathrm{CHVC}$ is only $1^{\circ}$ and the maximum 2.2 .

between $T_{\text {peak }}=0.20 \mathrm{~K}$ and $0.45 \mathrm{~K}$, the LDS results recovered $83 \%$ of the clouds found in the HIPASS data. The completeness of the LDS catalog drops rapidly at lower values of the peak temperature: HIPASS clouds less bright than $0.20 \mathrm{~K}$ are almost completely absent from the LDS catalog. The incompleteness at low peak temperatures will be more important for the smaller CHVCs than for the somewhat less compact CHVC:s. Some of the smaller objects have a total column density which is difficult to distinguish from the LDS spectral noise (see Sect. 3.2). Their FWHM angular sizes are less than $25^{\prime}$ and they may be centered as much as $10^{\prime}$ from the nearest LDS telescope pointing. The small sizes of the CHVCs and the less-than-Nyquist sampling interval of the LDS can conspire to result in an observation with a lower signal-to-noise ratio in the LDS than in the HIPASS.

The differences in sensitivity not only influence the number of sources that are detected, but also the way in which they are classified. Within the zone of overlapping declinations, 33 CHVCs were found in the HIPASS material with a peak temperature above $0.20 \mathrm{~K}$. Of these 33 sources, 19 were also found in the LDS. The appearance of these 19 sources in the LDS led 7 of them to be classified as CHVCs, 7 as CHVC:s, and the remaining 5 as HVCs. The difference in assignment is primarily due to the differences in the properties of the two sets of data. The possibility of a different designation is greater for the fainter sources. For the eight sources with a HIPASS peak temperature above $0.35 \mathrm{~K}$, four have a different LDS designation, whereas there is only agreement for three of the eleven sources which are fainter than $0.35 \mathrm{~K}$. Evidently the differing sensitivities to compact and extended structures of the LDS and
HIPASS do not allow for consistent classification of the weakest objects. Figure 15 shows, as an example, the HIPASS data for a cloud which is seen in projection against an extended filament: the LDS data were unable to detect the weak emission of the filament, and as a consequence the search algorithm applied to the LDS returned a CHVC designation. This example demonstrates that the classification of clouds depends on the sensitivity of the survey.

If the HIPASS and LDS catalogs are compared or if they are used together, for example to investigate the all-sky properties of the compact-object ensemble, then due attention should be given to the higher expected detection rate in the southern material, and to its greater sensitivity to the most compact features. A straightforward merger of the two catalogs would neglect the higher rate of detections in the southern hemisphere and the possibly different designation of a limited number of the clouds. To correct for the higher detection rate in the south, Table 3 can be used to roughly estimate the likelihood that a given cloud which is observed in the HIPASS data also will be observed in the LDS.

\subsection{Completeness and homogeneity of the LDS sample of CHVCs}

Before discussing some aspects of the LDS sample of CHVCs, a few additional remarks concerning completeness and homogeneity of the sample are in order. These parameters are influenced by the non-Nyquist sampling of the LDS and its finite sensitivity, 

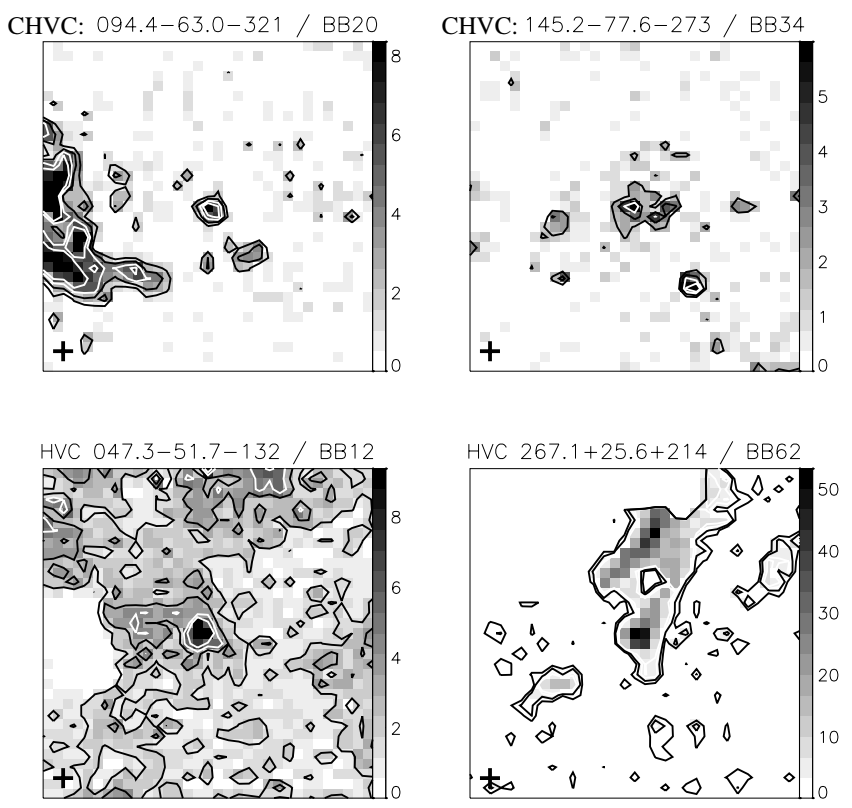

Fig. 14. Examples of objects which were identified as CHVCs by BB99, but which have been re-classified following the more stringent criteria described in this paper, which measures the isolation of a feature using the $N_{\mathrm{HI}}$ contour at a fixed level of $1.5 \times 10^{18} \mathrm{~cm}^{-2}$, rather than at the level of $50 \%$ of the peak in each object as used by BB99. The features shown in the upper two panels were reclassified as CHVC:s, thus as possible members of the class of compact objects; but the features shown in the two lower panels are not isolated according to the new criterion, and were reclassified as HVCs. The cross in the lower left of each panel indicates the angular extent of a true degree on the sky. The contours are drawn at 1.5, 3, 4.5 and $6 \times 10^{18} \mathrm{~cm}^{-2}$; the intensity scale is indicated by the color bar, in units of $\mathrm{K} \mathrm{km} \mathrm{s}^{-1}$.

Due to the finite sensitivity of the LDS, we will have missed clouds with peak temperatures or column densities below threshold values. After converting the HIPASS peak temperatures in Table 3 to the LDS temperature scale, that table can be used to estimate the number of undetected features. A comparison of the objects with detections both in the HIPASS and in the LDS listings shows that the average of the ratio between the HIPASS and LDS peak temperatures equals 1.5. This difference can be understood in terms of the differing angular sampling intervals and resolutions of the two surveys.

The validity of Fig. 16 as an indication of completeness is suggested by the detection rate for known external galaxies which appear in the LDS. Hartmann \& Burton (1997) list all of the galaxies that are cataloged in LEDA and detected in the LDS. Of the known external galaxies with $\left|V_{\mathrm{DEV}}\right|>$ $70 \mathrm{~km} \mathrm{~s}^{-1}$ and $V_{\mathrm{LSR}}<350 \mathrm{~km} \mathrm{~s}^{-1}$, all those with a peak $\mathrm{H}_{\mathrm{I}}$ brightness temperature greater than $0.13 \mathrm{~K}$ were found by the search algorithm. Of the 12 galaxies with $0.09 \mathrm{~K} \leq T_{\text {peak }}<$ $0.12 \mathrm{~K}, 42 \%$ were found. The temperatures and therefore sensitivities were measured at the grid points of the survey; point sources that are not located at a grid point will have been observed with a reduced sensitivity, which depends on the telescope beam and the distance to the nearest grid point. The LDS sampled the sky on a 0.5 by 0.5 lattice; the sensitivity away
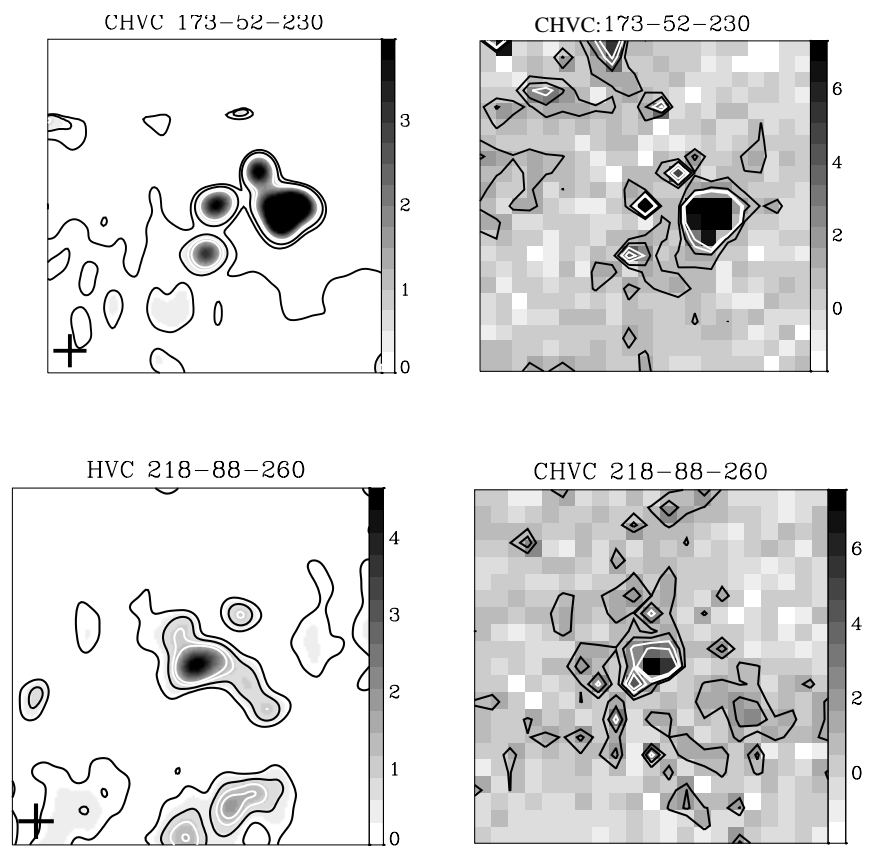

Fig. 15. Velocity-integrated images of two objects which were retrieved by the search algorithm both from the HIPASS material as reported by Putman et al. (2002) and from the LDS material discussed here, but which were classified differently by the search algorithm because of the differing observational parameters of the two surveys. The images show the HIPASS data on the left after spatial smoothing to 36 arcmin FWHM; the LDS, on the right. The angular orientation and the velocity range of integration are the same for both of the paired images. For the object represented in the upper pair, a classification of CHVC followed from the HIPASS material, but a classification of CHVC: from the LDS data. The object represented in the lower pair of images was classified as a HVC from the HIPASS material but as CHVC from the LDS. The cross in the lower left of each panel indicates the angular extent of a true degree on the sky. The contours are drawn at $N_{\mathrm{HI}}=1.5,3,4.5$ and $6 \times 10^{18} \mathrm{~cm}^{-2}$; the intensity scale is indicated by the color bar, in units of $\mathrm{K} \mathrm{km} \mathrm{s}^{-1}$.

from the grid points falls off as a Gaussian with a FWHP of $36^{\prime}$. The results, shown in Fig. 16, lead us to consider the LDS catalog to be $100 \%$ complete for point sources with $T_{\text {peak }} \geq 0.3 \mathrm{~K}$. The solid curve follows the trend as indicated by the HIPASS catalog.

Objects with $\mathrm{H}$ I properties like the CHVCs will be missed if their emission is superposed on emission from intermediateor high-velocity complexes, or from our Galaxy. Independent of their exact origin, it is likely that some of these clouds will coincide with extended emission of a different type. Related to this problem is the selection based on the deviation velocity of the clouds. We mentioned above that $12 \%$ of the BB99 sample were excluded from this study by this selection criterion. Before we can properly investigate the exact influence of the obscurations or exclusion of some parts of the LDS, we have to model the intrinsic distribution of the CHVCs and then assess how they would appear in the data set. We investigate this issue in our analysis of the all-sky distribution of CHVCs (de Heij et al. 2002). 
Table 3. Number of clouds classified as CHVCs and CHVC:s in the overlap zone of the HIPASS and LDS surveys, listed according to the peak brightness temperatures of the clouds. The data from the two surveys differ sufficiently that the search algorithm described here may have returned a different classification from the HIPASS material than from the LDS. The peak temperatures refer to the HIPASS temperature calibration. The statistics show that the LDS catalog of compact anomalous-velocity objects is likely to be complete for clouds with $T_{\text {peak }}>0.3 \mathrm{~K}$, within the velocity range constrained by the bandwidth of the survey and by the adapted deviation velocity.

\begin{tabular}{|c|c|c|}
\hline \hline \multirow{2}{*}{$T_{\text {peak }}$} & \multicolumn{2}{|c|}{ \# CHVCs + CHVC:s } \\
\cline { 2 - 3 }$(\mathrm{K})$ & HIPASS & LDS \\
\hline $0.10 \ldots 0.15$ & 28 & 0 \\
$0.15 \ldots 0.20$ & 26 & 4 \\
$0.20 \ldots 0.25$ & 17 & 7 \\
$0.25 \ldots 0.30$ & 8 & 5 \\
$0.30 \ldots 0.35$ & 10 & 9 \\
$0.35 \ldots 0.40$ & 8 & 7 \\
$0.40 \ldots 0.45$ & 2 & 1 \\
$0.45 \ldots 0.50$ & 3 & 3 \\
$0.50 \ldots 0.55$ & 1 & 1 \\
$0.55 \ldots 0.60$ & 3 & 3 \\
\hline
\end{tabular}

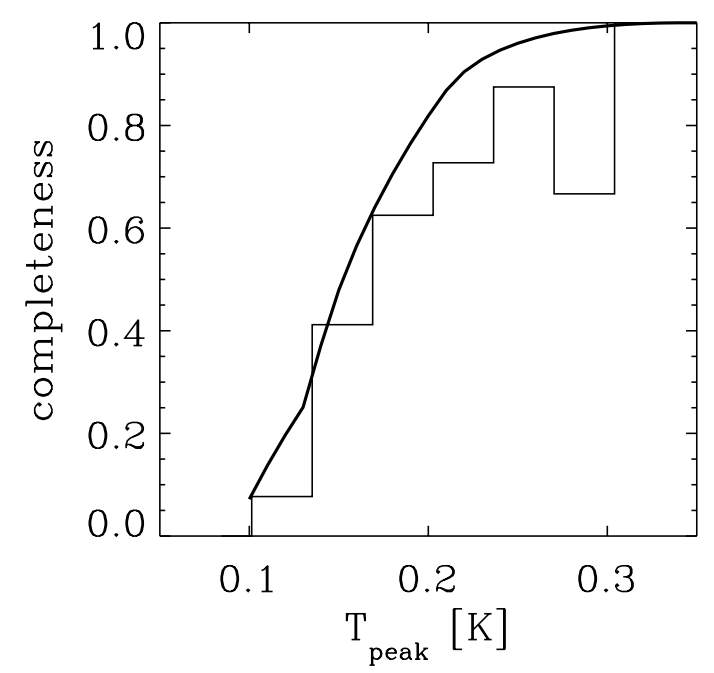

Fig. 16. Degree of completeness expected from the application of the search algorithm for point sources with given temperature. The fulldrawn line shows the expected completeness based on comparison between the results of our search and a catalog of nearby galaxies extracted from LEDA and the LDS. The histogram shows, for objects lying at declinations in the region of overlap of the HIPASS and LDS survey, the fraction of CHVCs which are listed both in the HIPASS catalog of Putman et al. (2002) and in the LDS catalog of Table 2. The histogram follows Table 3, after transforming the HIPASS temperatures to the LDS scale. The average ratio of the HIPASS and LDS peak temperature for a given cloud is not unity (but equals 1.5), due to the differences in the spectral and spatial resolutions of the two surveys.

Although the part of the LDS that was searched only covered the sky within the velocity interval $V_{\mathrm{LSR}}=-450 \mathrm{~km} \mathrm{~s}^{-1}$ to $+350 \mathrm{~km} \mathrm{~s}^{-1}$, there are indications that we do not miss many (if any) clouds because of the velocity interval. The highvelocity feature with the most extreme negative velocity yet found is HVC 110.6-07.0-466, discoverd by Hulsbosch (1978) and subject to substantial subsequent observation as referenced in Table 2. The Wakker \& van Woerden tabulation, which relied on survey data covering the range $-900 \mathrm{~km} \mathrm{~s}^{-1}$ to $+750 \mathrm{~km} \mathrm{~s}^{-1}$, found no high-velocity cloud at a more negative velocity. The HIPASS search reported by Putman et al. (2002) sought anomalous-velocity emission over the range $-700<V$ LSR $<+1000 \mathrm{~km} \mathrm{~s}^{-1}$. Of the 194 HIPASS CHVCs cataloged by Putman et al., ten have $V_{\mathrm{LSR}}<-300$, but the most extreme negative velocity is $-353 \mathrm{~km} \mathrm{~s}^{-1}$. This CHVC, namely CHVC $125.1-66.4-353$ occurs, not surprisingly, in the quadrant where the northern data shows a preference for extreme negative velocities.

The north/south kinematic asymmetries are a well-known property of the anomalous-velocity H I. In terms of the Local Group deployment discussed by Blitz et al. (1999) and by BB99, the most extreme negative velocities would be found in the general region of the barycenter of the Local Group, whereas the most extreme positive LSR velocities, which would be more modest in amplitude than the extreme negative velocities, would be found in the general region of the anti-barycenter of the Local Group. The LDS does not reach low enough declinations to embrace the anti-barycenter region, although the feature in Table 2 with the highest positive velocity, CHVC: $357.5+05.6+268$ is well removed from the direction of the barycenter ${ }^{1}$. Of the 194 CHVCs in the HIPASS catalog, only 7 have $V_{\mathrm{LSR}}$ greater than $+300 \mathrm{~km} \mathrm{~s}^{-1}$, and only one has a velocity greater than $350 \mathrm{~km} \mathrm{~s}^{-1}$, namely CHVC 258.2 - 23.9+359. All of the seven CHVCs with substantial positive velocities lie deep in the third longitude quadrant, or in the fourth; the mean longitude of these seven CHVCs is $278^{\circ}, 185^{\circ}$ removed from the longitude appropriate for the solar apex motion in the Local Group Standard of Rest reference frame as determined by Karachentsev \& Makorov (1996), and which thus roughly corresponds with the direction of the Local Group anti-barycenter. The Wakker \& van Woerden compilation lists no high-velocity feature with a more positive velocity than that of their HVC $305.0-10.0+312$, also in the general direction preferentially represented by positive velocites.

In view of these detection statistics, we consider it unlikely that the velocity range of the LDS has caused a significant number of features to be missed at the declinations observed. In other words, the true velocity extent, as well as the non-zero mean in the LSR frame, of the anomalous-velocity ensemble

\footnotetext{
1 Although none of the compact $\mathrm{H}$ i clouds listed in Table 2 showed an obvious optical counterpart in our perusal of the DSS, it is not yet firmly ruled out that the listing might be contaminated by an obscured nearby galaxy, as yet undetected at optical or infrared wavelengths. Two of the features listed in our catalog appear particularly suspicious in this regard, namely CHVC: $357.5+05.6+268$ and CHVC 004. $4+05.7+202$. Both are located along sight lines traversing the Galactic Bulge, and thus lie in directions of exceptionally high optical obscuration, and both deviate strongly from the kinematic patterns defined by the other members of the ensemble. This deviation is particularly evident in Fig. 17, a figure of the sort frequently used to establish membership in the Local Group.
} 


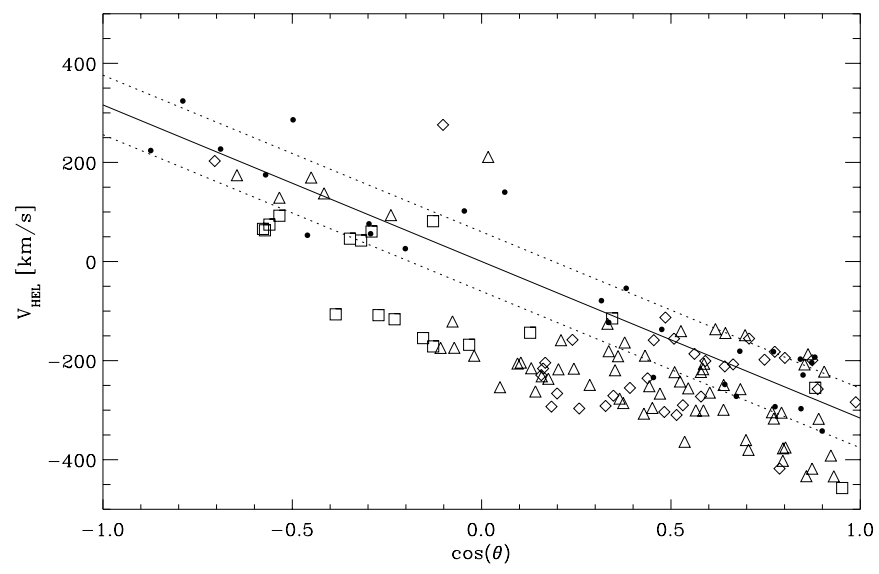

Fig. 17. Heliocentric velocities of nearby galaxies and of the ensemble of compact high-velocity clouds, plotted as a function of $\cos (\theta)$, where $\theta$ is the angle between the object and the location of the apex of the solar motion relative to the center of mass of the Local Group. CHVCs are shown as triangles, and the less-constrained CHVC: and CHVC? objects as diamonds and squares, respectively; the Local Group galaxies, from the tabulation of Mateo (1998), are plotted as filled circles. The solid line represents the solar motion of $V_{\odot}=316 \mathrm{~km} \mathrm{~s}^{-1}$ toward $l=93^{\circ}, b=-4^{\circ}$ as determined by Karachentsev \& Markarov (1996). The dashed lines show the envelope one standard deviation $\left( \pm 60 \mathrm{~km} \mathrm{~s}^{-1}\right)$ about the $V_{\mathrm{HEL}}, \cos (\theta)$ relation, pertaining for galaxies considered firmly established as members of the Local Group.

appear well represented by the extrema of $-466 \mathrm{~km} \mathrm{~s}^{-1}$ and $+359 \mathrm{~km} \mathrm{~s}^{-1}$.

\section{Discussion}

\subsection{What defines a CHVC?}

The concept of a distinct class of compact, isolated highvelocity clouds has emerged from the visual inspection of large area images of good sensitivity and spatial sampling like the LDS in the north and the HIPASS in the south. There appears to be a class of high-contrast features which are at best only marginally resolved with half degree angular resolution and that can not easily be distinguished from the $\mathrm{H}_{\mathrm{I}}$ signature of a low mass external galaxy. The Hi signature of an external galaxy in the LDS (see Fig. 15 of Hartmann \& Burton 1997) is a moderately high peak column density of a few times $10^{19} \mathrm{~cm}^{-2}$ or more (averaged over the 36 arcmin beam), an H I FWHM linewidth varying between 20 and $375 \mathrm{~km} \mathrm{~s}^{-1}$ commensurate with the galaxy mass and inclination, and a sharply bounded angular extent, such that emission at column densities above a few times $10^{18} \mathrm{~cm}^{-2}$ is confined to less than about $2^{\circ}$ diameter.

This last fact is of particular physical relevance, since the precipitous decline in $\mathrm{H}_{\mathrm{I}}$ column density seen at the edges of nearby galaxies can be understood as arising from photoionization due to the intergalactic radiation field. Detailed studies of individual systems (NGC 3198 by Maloney 1993; M 33 by Corbelli \& Salpeter 1993) show that an exponential decline in neutral column density, with a scale-length of about $1 \mathrm{kpc}$ sets in below a critical column density of about $2 \times 10^{19} \mathrm{~cm}^{-2}$.
This neutral column density is comparable to the Warm Neutral Medium layer (with a temperature of about $8000 \mathrm{~K}$ ) required to provide sufficient shielding from UV and soft X-ray radiation such that condensation of Cool Neutral Medium clumps (with temperature of about $100 \mathrm{~K}$ ) can take place (e.g. Wolfire et al. 1995a) for ambient thermal pressures comparable to, or less than, those found in the solar neighbourhood. The picture that emerges is a nested structure of CNM cores, shielded by WNM cocoons and surrounded by a Warm Ionized Medium halo. For nearby galaxies the WNM cocoon at the edge of the gaseous disk has an observed exponential scale-length of about $1 \mathrm{kpc}$ (Maloney 1993; Corbelli \& Salpeter 1993). The exponential scale-length of the partially ionized WNM cocoon is determined by both the total column density distribution and the incident ionizing spectrum. For smooth distributions of total column density and a wide range of power-law spectral indices, Corbelli \& Salpeter (1993) find neutral scale-lengths of about $1 \mathrm{kpc}$. The reason that this transition zone is so much more extended than in a classical $\mathrm{H}_{\text {II }}$ region is found in the wide range of photon energies, and hence penetrating depths, of the ionizing spectrum.

It is not obvious that all of these considerations need apply to the population of high-velocity clouds. If the HVCs are near the Galactic disk, then they will be subject to severe tidal distortions of their intrinsic gas distributions and be exposed to variable ionizing radiation levels depending on local circumstances. Due to changing physical conditions it is conceivable that thermal and pressure equilibrium may not be achieved. The requirements for achieving such equilibrium have been considered by Wolfire et al. (1995b), who conclude that even for heights above the Galactic plane of only $3 \mathrm{kpc}$, thermal equilibrium should be marginally achieved within the WNM and easily within the CNM at an infall velocity as high as $150 \mathrm{~km} \mathrm{~s}^{-1}$. At larger distances the requirements that the thermal timescale be shorter than the time to experience significant pressure variations are even more easily satisfied. It thus seems reasonable to expect that high-velocity clouds might also be described by CNM clumps within a WNM shielding cocoon surrounded by WIM halos. If such objects occur at distances greater than about $60 \mathrm{kpc}$, tidal effects would be less disruptive, the UV radiation field would be more nearly isotropic, and an $\mathrm{H}_{\mathrm{I}}$ concentration of about $2 \mathrm{kpc}$ diameter would have an angular size of less than $2^{\circ}$.

\subsection{Application of a digital filter to the LDS}

Motivated by the distinctive visual appearance of sharply bounded $\mathrm{HI}_{\mathrm{I}}$ peaks in the LDS data, we have considered whether a simple digital filtering might not serve to isolate a subset of these features from the general Hi emission of the Galaxy and the HVC complexes, and in so doing offer independent confirmation of the results achieved with the procedure outlined in Sect. 3. We considered a two-dimensional discrete derivative convolving kernel, consisting of a delta function at the origin together with a negative ring of unit integral at a radius of $1^{\circ}$. After evaluating the convolution of each channel 
map with this kernel, we normalize the result by the original intensity, wherever this exceeds some minimum significance:

$$
F(x, y)=\frac{1}{I} \frac{\mathrm{d} I}{\mathrm{~d} r}
$$

for $I>n \sigma$. As an illustration, this filter was applied to an Aitoff-projected cube of the LDS data centered at $(l, b)=$ $\left(123^{\circ}, 0^{\circ}\right)$ after a velocity smoothing to $25 \mathrm{~km} \mathrm{~s}^{-1} F W H M$, and restricted to intensities exceeding $10 \sigma$, corresponding to $0.14 \mathrm{~K}$ brightness. The peak filtered response was then determined along each spectrum; this is illustrated as the greyscale in Fig. 18 for values exceeding 0.85 in units of the normalized derivative. Overlaid on the peak filter values are square symbols at the positions of 59 cataloged external galaxies. Of these 59 nearby $\mathrm{HI-emitting} \mathrm{galaxies,} 57$ are listed in Table 4 of Hartmann \& Burton (1997); the remaining two are Dwingeloo 1, discovered by Kraan-Korteweg et al. (1994), and Cepheus 1, discovered by Burton et al. (1999). Open circles in this figure show the positions of the $116 \mathrm{CHVCs}$ which we catalog here; crosses show the positions of 72 CHVC candidates which were not confirmed in subsequent observations, and triangles show the positions of $84 \mathrm{CHVC}$ candidates for which no confirming observations have yet been obtained.

It is striking how the vast majority of both normal Galactic and HVC complex emission has been eliminated by this simple filtering. About half of the peaks returned by the normalized derivative filter have an over-plotted symbol corresponding either to a cataloged external galaxy or to a CHVC. The 18 cataloged galaxies and 39 CHVCs which do not have a strong filter response can all be understood in terms of a poorly matched velocity width (since objects well-matched with a $25 \mathrm{~km} \mathrm{~s}^{-1} F W H M$ were selected in this case) or a very low peak brightness (that is, below the cut-off of $0.14 \mathrm{~K}$ ). In addition to the filter peaks with over-plotted symbols there are a similar number, about 140 peaks, exceeding 0.85 in the normalized spatial derivative without an overplotted identification. Closer inspection of these additional filter peaks reveals that they all correspond to sub-structure within more extended HVC complexes. These are quite distinct from the CHVCs in that they are not sharply bounded at a column density of $1.5 \times 10^{18} \mathrm{~cm}^{-2}$, but instead are connected (at least in projected position-velocity space) to more extended HVC complexes at a higher column density.

\subsection{Brief remarks on the spatial and kinematic deployment of the northern CHVCs}

In a separate paper (de Heij et al. 2002), we analyse the LDS CHVC sample merged with the sample identified by Putman et al. (2002) in the southern hemisphere HIPASS material. Here we briefly note the global properties of the compact objects found in the LDS.

Figure 18 shows the distribution on the sky of the compact, isolated objects cataloged in Table 2. The objects are distributed rather uniformly across the northern sky. In particular, there is no clear sign of a preference in the sky distribution either for the Galactic disk, or for most of the known high-velocity cloud complexes, or the Magellanic Stream.
One exception to this general conclusion is an apparent concentration centered near $(l, b)=(25,-30)$, a region which Wakker \& van Woerden (1991) identify with their Galactic Center Negative Velocity Population of HVCs. This region appears to be particularly rich in compact objects statisfying very stringent requirements for isolation in column density. For comparison, the distribution of all nearby galaxies detected in the LDS data is also plotted in the figure.

Figure 19 shows the velocities of all of the compact clouds cataloged in Table 2, calculated for three different reference frames and plotted as function of the Galactic longitude. The velocity dispersion of the sample decreases from $174 \mathrm{~km} \mathrm{~s}^{-1}$, to $101 \mathrm{~km} \mathrm{~s}^{-1}$, and then to $97 \mathrm{~km} \mathrm{~s}^{-1}$, in going from the reference frame of the Local Standard of Rest, to that of the Galactic Standard of Rest, and then to that of the Local Group Standard of Rest. Selection effects related to obscuration by Galactic emission at low deviation velocities may be very important in determining the apparent velocity dispersion in the various reference frames. The average velocity changes from $-191 \mathrm{~km} \mathrm{~s}^{-1}$, to $-127 \mathrm{~km} \mathrm{~s}^{-1}$, and then to $-117 \mathrm{~km} \mathrm{~s}^{-1}$, for the three different reference frames, respectively. Although the differences between the Galactic and Local Group system are not very large, the values are lower in the Local Group coordinate system. Inclusion of the data of the southern hemisphere in the analysis has to indicate if the small difference is significant or not. The difference of $10 \mathrm{~km} \mathrm{~s}^{-1}$ between the velocity dispersion in the LGSR reference frame found by BB99 and that found here is probably due to the differences in the samples used, with the BB99 one being smaller.

\subsection{Summary and conclusions}

An automated procedure has been developed to extract anomalous-velocity clouds from $\mathrm{H}_{\mathrm{I}}$ survey material. We have applied the algorithm to the Leiden/Dwingeloo survey and catalog the properties of a total of 917 HVC features with a deviation velocity in excess of $70 \mathrm{~km} \mathrm{~s}^{-1}$. Since the algorithm requires the existence of a local maximum in position-velocity which is distinct from the bulk of Galactic emission, the catalog can not be usefully extended to include the intermediate velocity clouds which are strongly blended with the Galaxy. We have searched our HVC catalog for all isolated clouds, defined by having a lowest significant column density contour $\left(3 \sigma \sim 1.5 \times 10^{18} \mathrm{~cm}^{-2}\right)$ which is (1) closed, with its greatest radial extent less than $10^{\circ}$ by $10^{\circ}$; and (2) not confused by the presence of nearby extended emission. A total of $116 \mathrm{ob}$ jects have been tabulated which at least partially satisfy these criteria. Independent confirmation is available for all of these clouds, some of which appear in only single spectra in the LDS. Of the 116 clouds, 54 had been identified as CHVCs by Braun \& Burton (1999), some others had been detected in earlier work referenced in the catalog but are confirmed as isolated by the data and analysis presented here, and others are reported here for the first time.

Although objects as large as 7 or $8^{\circ}$ were permitted by our selection criteria, the resulting distribution is strongly peaked at a median value of $1^{\circ} \mathrm{FWHM}$ and has a maximum observed 


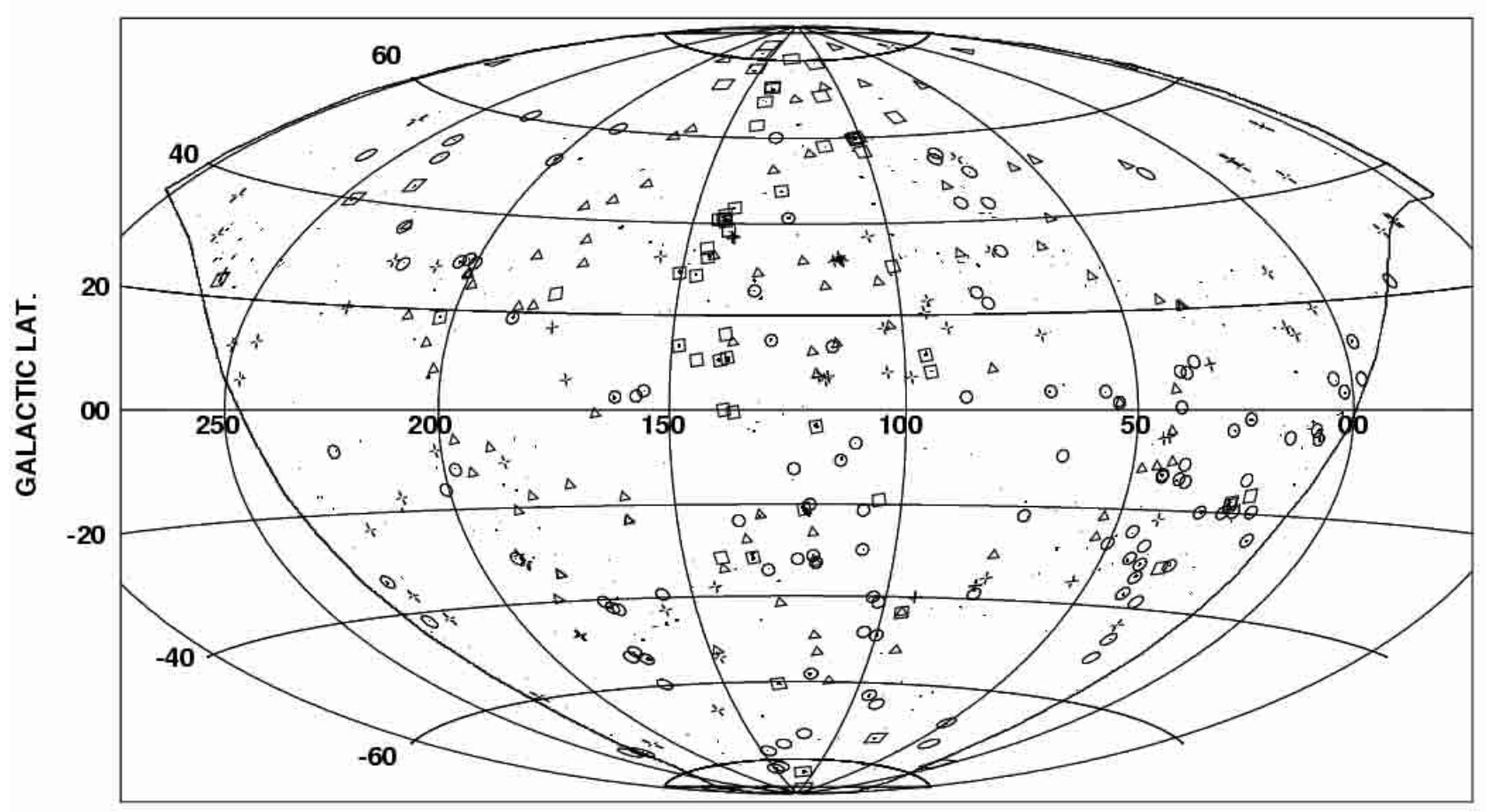

GALACTIC LONG.

Fig. 18. Distribution on the sky of the compact anomalous-velocity objects (CHVCs) found by application of the search algorithm and selection criteria to the Leiden/Dwingeloo Survey. The background greyscale is the output of a spatial derivative filter to the LDS data (matched to the median cloud linewidth and angular size) where this exceeds 0.85 in units of the normalized derivative. Overlaid are square symbols at the positions of 59 cataloged external galaxies, open circles for the $116 \mathrm{CHVCs}$ which we catalog here; crosses at the positions of $72 \mathrm{CHVC}$ candidates which were not confirmed in subsequent observations, and triangles at the positions of 84 CHVC candidates for which no confirming observations have yet been obtained. The CHVCs are not strongly clumped in the northern sky; and in particular show no concentration toward the extended HVC complexes or Magellanic Stream but do show some concentration toward the region near $(l, b)=\left(25^{\circ},-30^{\circ}\right)$ which has previously been called the Galactic Center Negative velocity population. The solid curve indicates the nominal $\delta=-30^{\circ}$ cut-off of the LDS coverage.

diameter of only $2^{\circ} 2$. Isolated HVCs are observed to be relatively compact. Conversely, although the well known HVC complexes exhibit a wealth of small-scale structure that is comparable in angular scale $\left(\sim 1^{\circ}\right)$ these structures are not isolated. The local maxima within the HVC complexes are surrounded by extended emission with column densities in the range $5-20 \times 10^{18} \mathrm{~cm}^{-2}$.

The significance of high galactic latitude $\mathrm{H}_{\mathrm{I}}$ features which are isolated in column density down to a level as low as $1.5 \times 10^{18} \mathrm{~cm}^{-2}$, is that this is about an order of magnitude lower than the critical column density identified at the edges of nearby galaxies (Maloney 1993; Corbelli \& Salpeter 1993), $\sim 2 \times 10^{19} \mathrm{~cm}^{-2}$, where the ionized fraction is thought to increase dramatically due to the extragalactic radiation field. Unless very contrived geometries are invoked of some unseen population of high column density absorbers, these objects will need to provide their own shielding to ionizing radiation. (This point has also been made by Hoffman et al. 2002.) Selfconsistent calculations of the ionization balance in the shielding layer exposed to a power-law extragalactic ionizing photon field yield a typical $\mathrm{H}_{\mathrm{I}}$ exponential scale-length of $1 \mathrm{kpc}$ (Corbelli \& Salpeter 1993). The small median angular size of the CHVCs, of about $1^{\circ} F W H M$, might then imply substantial distances, greater than about $120 \mathrm{kpc}$.

The kinematic and spatial deployment of the enlarged sample shows that both the Galactic Standard of Rest and the Local Group Standard of Rest frames substantially lower the velocity dispersion of the population. This is consistent with the hypothesis that the CHVCs are a distant component of the highvelocity cloud phenomenon, at substantial distances and with a net in-fall towards the Galaxy or the Local Group barycenter. However, the velocity dispersion of the sample is likely to be very strongly affected by selection effects related to Galactic obscuration of objects at low deviation velocity.

A more complete analysis of the kinematic and spatial deployment of the all-sky CHVC ensemble incoporates both the LDS sample and the results of a comparable search by Putman et al. (2002) in the HIPASS data, and is reported separately by de Heij et al. (2002).

Acknowledgements. The Westerbork Synthesis Radio Telescope is operated by the Netherlands Foundation for Research in Astronomy, under contract with the Netherlands Organization for Scientific Research. 


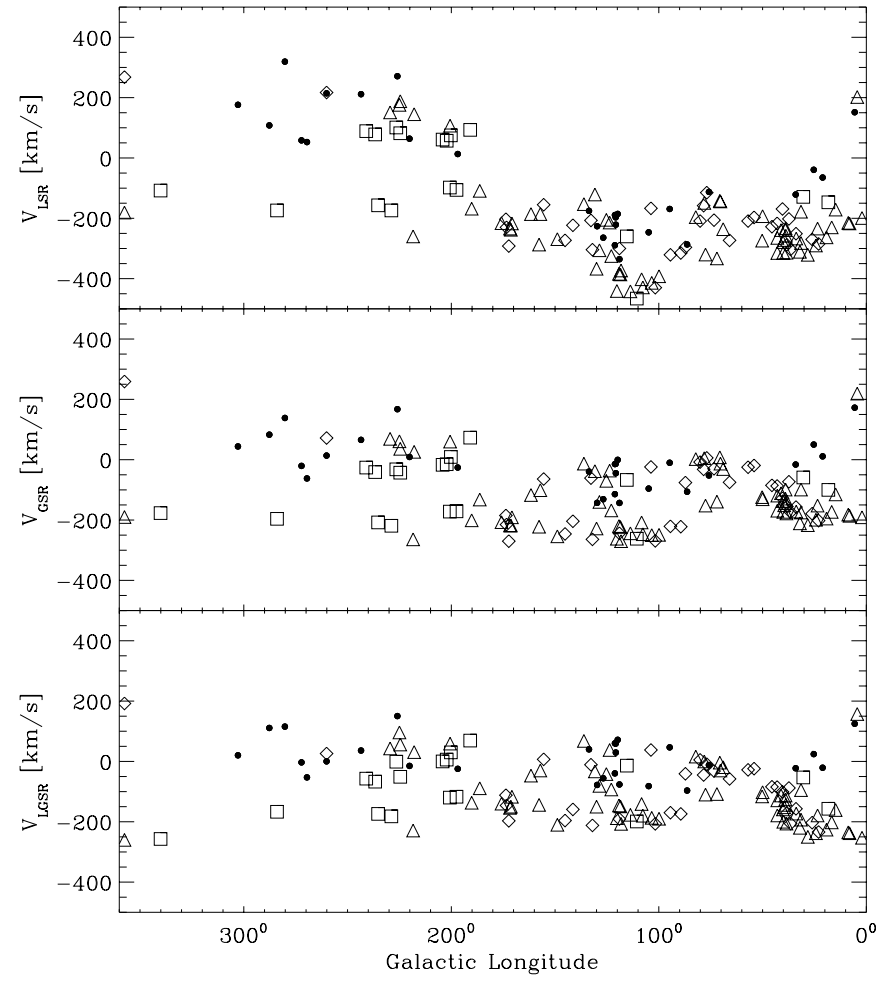

Fig. 19. Kinematic deployment of the compact-object sample plotted as a function of the galactic longitude for three different kinematic reference frames, compared with the kinematic distribution of Local Group galaxies. The CHVCs are shown as triangles; the lessconstrained CHVC: and CHVC? objects as diamonds and squares, respectively. The kinematic distribution of the Local Group galaxies tabulated by Mateo (1998) is traced by the filled circles. There is a decrease in the average velocity of the compact-object ensemble, as well as in the velocity dispersion, when progressing from the Local Standard of Rest system (upper panel), to the Galactic Standard of Rest system (middle panel), to the Local Group Standard of Rest system (lower panel).

\section{References}

Arp, H. 1985, AJ, 90, 1012

Bajaja, E., Cappa de Nicolau, C. E., Cersosimo, J. C., et al. 1985, ApJS, 58, 143

Bajaja, E., Morras, R., \& Pöppel W. G. L. 1987, Pub. Astr. Inst. Czech. Ac. Sci., 69, 237

Baker, P. L., \& Burton, W. B. 1975, ApJ, 198, 281

Barnes, D. G., Staveley-Smith, L., de Blok, W. J. G., et al. 2001, MNRAS, 322, 486

Binney, J., \& Merrifield, M. 1998, Galactic Astronomy (Princeton University Press)

Blitz, L., Spergel, D. N., Teuben, P. J., Hartmann, D., \& Burton, W. B. 1999, ApJ, 514, 818

Braun, R., \& Burton, W. B. 1999, A\&A, 341, 437 (BB99)

Braun, R., \& Burton, W. B. 2000, A\&A, 354, 853

Brüns, C., Kerp, J., \& Pagels, A. 2001, A\&A, 371, 816

Burton, W. B., Braun, R., \& Chengalur, J. N. 2001, A\&A, 369, 616 (erratum in A\&A, 375, 227)
Burton, W. B., Braun, R., Walterbos, R. A. M., \& Hoopes, C. G. 1999, AJ, 117, 194

Cohen, R. J., \& Mirabel, I. F. 1979, MNRAS, 186, 217

Corbelli, E., \& Salpeter, E. E. 1993, ApJ, 419, 104

Davies, R. D. 1975, MNRAS, 170, 45P

de Heij, V., Braun, R., \& Burton, W. B. 2002, A\&A, in press [astro-ph/0206306]

Eichler, D. 1976, ApJ, 208, 694

Einasto, J., Haud, U., Jôeveer, M., \& Kaasik, A. 1976, MNRAS, 177, 357

Giovanelli, R. 1981, AJ, 86, 1468

Hartmann, D. 1994, Ph.D. Thesis, University of Leiden

Hartmann, D., Kalberla, P. M. W., Burton, W. B., \& Mebold, U. 1996, A\&AS, 119, 115

Hartmann, D., \& Burton, W. B. 1997, Atlas of Galactic Neutral Hydrogen (Cambridge University Press)

Henning, P. A. 1992, ApJS, 78, 365

Hoffman, G. L., Salpeter, E. E., \& Pocceschi, M. G. 2002, ApJ, in press [astro-ph/0205404]

Hulsbosch, A. N. M. 1978, A\&A, 66, L5

Hulsbosch, A. N. M., \& Wakker, B. P. 1988, A\&AS, 75, 191

Karachentsev, I. D., \& Makarov, D. A., 1996, AJ, 111, 794

Kraan-Korteweg, R. C., Loan, A. J., Burton, W. B., et al. 1994, Nature, 372, 77

Maloney, P. 1993, ApJ, 414, 41

Mateo, M. 1998, ARA\&A, 36, 435

Mirabel, I. F. 1981, ApJ, 247, 97

Mirabel, I. F., \& Cohen, R. J. 1981, MNRAS, 188, 219

Muller, C. A., Oort, J. H., \& Raimond, E. 1963, C. R. Acad. Sci. Paris, 257,1661

Oort, J. H. 1966, Bull. Astr. Inst. Netherlands, 18, 421

Oort, J. H. 1970, A\&A, 7, 381

Oort, J. H. 1981, A\&A, 94, 359

Putman, M., \& Gibson, B. K. 1999, PASA, 16, 70

Putman, M., de Heij, V., Staveley-Smith, L., et al. 2002, AJ, 123, 873

Saraber, M. J. M., \& Shane, W. W. 1974, A\&A, 30, 365

Stutzki, J., \& Güsten, R. 1990, ApJ, 356, 513

Thilker, D. A., Braun, R., \& Walterbos, R. A. M. 1998, A\&A, 332, 429

van Woerden, H., Schwarz, U. J., Peletier, R. F., Wakker, B. P., \& Kalberla, P. M. W. 1999, Nature, 400, 138

Verschuur, G. L. 1975, ARA\&A, 13, 257

Voskes, T., \& Burton, W. B. 1999, in New Perspectives on the Interstellar Medium, ed. A. R. Taylor, T. L. Landecker, \& G. Joncas, ASP Conf. Ser., 168375

Wakker, B. P. 1990, Ph.D. Thesis, University of Groningen

Wakker, B. P., 2001, ApJS, 136, 463

Wakker, B. P., \& van Woerden, H. 1991, A\&A, 250, 509

Wakker, B. P., \& van Woerden, H. 1997, ARA\&A, 35, 217

Wakker, B. P., van Woerden, H., \& Gibson, B. K. 1999, in Stromlo Workshop on High-Velocity Clouds, ed. B. K. Gibson \& M. E. Putman, ASP Conf. Ser., 166311

Williams, J. P., de Geus, E. J., \& Blitz, L. 1994, ApJ, 428, 693

Wolfire, M. G., Hollenbach, D., McKee, C. F., \& Bakes, E. L. O., 1995, ApJ, 443, 152

Wolfire, M. G., McKee, C. F., Hollenbach, D., \& Tielens, A. G. G. M., 1995, ApJ, 453, 673

Wright, M. C. H. 1979, ApJ, 233, 35 